\title{
Seniority in quantum many-body systems. I. Identical particles in a single shell
}

\author{
P. Van Isacker ${ }^{a}$, S. Heinze ${ }^{b}$ \\ ${ }^{a}$ Grand Accélérateur National d'Ions Lourds, CEA/DSM-CNRS/IN2P3, \\ BP 55027, F-14076 Caen Cedex 5, France \\ ${ }^{\mathrm{b}}$ Institut für Kernphysik der Universität zu Köln, 50937 Köln, Germany
}

\begin{abstract}
A discussion of the seniority quantum number in many-body systems is presented. The analysis is carried out for bosons and fermions simultaneously but is restricted to identical particles occupying a single shell. The emphasis of the paper is on the possibility of partial conservation of seniority which turns out to be a peculiar property of spin- $9 / 2$ fermions but prevalent in systems of interacting bosons of any spin. Partial conservation of seniority is at the basis of the existence of seniority isomers, frequently observed in semi-magic nuclei, and also gives rise to peculiar selection rules in one-nucleon transfer reactions.
\end{abstract}

Key words: quantum mechanics, many-body systems, seniority, nuclear shell model, interacting bosons

PACS: 03.65.Fd, 21.60.Cs, 21.60.Fw, 03.75.Mn

\section{Introduction}

The seniority quantum number was introduced by Racah for the classification of electrons in an $\ell^{N}$ configuration where it appears as a label additional to the total orbital angular momentum $L$, the total spin $S$ and the total angular momentum $J[1]$. About ten years after its introduction by Racah it was adopted in nuclear physics for the $j j$-coupling classification of nucleons in a single- $j$ shell $[2,3]$. Seniority refers to the number of particles that are not in pairs coupled to angular momentum $J=0$. The seniority quantum number is usually denoted by $v$, from the Hebrew word for seniority, 'vet(h)ek' [4,5]. In

Email address: isacker@ganil.fr (P. Van Isacker). 
nuclear physics the concept has proven extremely useful, especially in semimagic nuclei where only one type of nucleon (neutron or proton) is active and where seniority turns out to be conserved to a good approximation.

Seniority can be given a group-theoretical definition starting from the Lie algebra $\mathrm{U}(2 j+1)$ which contains all (infinitesimal) unitary transformations among the $2 j+1$ single-particle states $\left|j m_{j}\right\rangle$ with $m_{j}=-j,-j+1, \ldots,+j$, where $j$ is the angular momentum (henceforth referred to as spin) carried by the particle which is integer for bosons and half-odd-integer for fermions. A system of $N$ identical particles (i.e., no other internal degrees of freedom for the particles besides their spin) is characterized by the symmetric representation $[N]$ of $\mathrm{U}(2 j+1)$ in the case of bosons or the anti-symmetric representation $\left[1^{N}\right] \equiv[1,1, \ldots, 1]$ in the case of fermions. Seniority arises as a label $v$ associated with a subalgebra of $\mathrm{U}(2 j+1)$, either the orthogonal algebra $\mathrm{SO}(2 j+1)$, if $2 j+1$ is odd (bosons), or the (unitary) symplectic algebra $\operatorname{Sp}(2 j+1$ ), if $2 j+1$ is even (fermions). Both $\mathrm{SO}(2 j+1)$ and $\mathrm{Sp}(2 j+1)$ contain the rotation algebra as a subalgebra which shall be denoted as $\mathrm{SO}(3)$ and $\mathrm{SU}(2)$, respectively, to indicate that the total angular momentum $J$ must by integer for bosons whereas it can be integer or half-odd-integer for fermions, depending on $N$ being even or odd. Finally, any many-particle state is characterized by the projection $M_{J}$ of the total angular momentum $J$ associated with $\mathrm{SO}(2)$.

The seniority classification can be summarized as

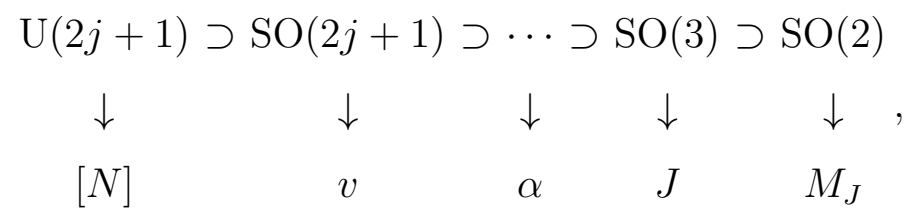

and

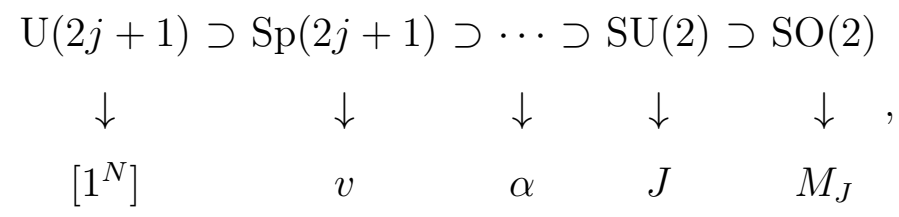

for bosons and fermions, respectively. In general, this classification is not complete, as indicated by the dots in the above equations. The allowed values of $v$ are $v=N, N-2, \ldots, 1$ or 0 , as can be obtained from the $\mathrm{U}(2 j+1) \supset \mathrm{SO}(2 j+1)$ or $\mathrm{U}(2 j+1) \supset \mathrm{Sp}(2 j+1)$ branching rules [6]. The allowed values of the total spin $J$ are obtained from the $\mathrm{SO}(2 j+1) \supset \mathrm{SO}(3)$ or $\mathrm{Sp}(2 j+1) \supset \mathrm{SU}(2)$ branching rules, which in general require a multiplicity label $\alpha$. Alternatively, seniority can be introduced via the quasi-spin formalism $[7,8]$ where it arises as a label associated with the Lie algebras $\mathrm{SU}(1,1)$ or $\mathrm{SU}(2)$ for bosons or 
fermions, respectively. This alternative definition is particularly valuable for generalizations towards different shells or several types of particles but it is not needed here.

This paper is concerned with the seniority classification of a system of $N$ identical particles with spin $j$ interacting through a general rotationally invariant two-body force. Symmetry arguments then dictate that the eigenstates of the hamiltonian carry good angular momentum $J$, as in the classifications (1) and (2). The central question addressed in this paper is what conditions are required for seniority $v$ to be a good quantum number for all or for part of the eigenstates.

To arrive at a more precise formulation of this question, let us introduce the following notation. A rotationally invariant two-body interaction $\hat{V}$ between the particles is specified by its $\lfloor j+1\rfloor$ matrix elements $\nu_{\lambda} \equiv\left\langle j^{2} ; \lambda m_{\lambda}|\hat{V}| j^{2} ; \lambda m_{\lambda}\right\rangle$ (where $\lfloor x\rfloor$ is the largest integer smaller than or equal to $x$ ). The notation $\left|j^{2} ; \lambda m_{\lambda}\right\rangle$ implies a normalized two-particle state with total angular momentum $\lambda$ and projection $m_{\lambda}$ which can take the values $\lambda=0,2, \ldots, 2 p$, $m_{\lambda}=-\lambda,-\lambda+1, \ldots,+\lambda$, where $2 p=2 j$ for bosons and $2 p=2 j-1$ for fermions. Since the interaction is rotationally invariant, there is no dependence on the label $m_{\lambda}$ which shall be suppressed henceforth. The interaction can then be written as $\hat{V}=\sum_{\lambda} \nu_{\lambda} \hat{V}_{\lambda}$ where $\hat{V}_{\lambda}$ is the operator defined through $\left\langle j^{2} ; \lambda^{\prime}\left|\hat{V}_{\lambda}\right| j^{2} ; \lambda^{\prime \prime}\right\rangle=\delta_{\lambda \lambda^{\prime}} \delta_{\lambda \lambda^{\prime \prime}}$

A precise formulation can now be given of the question that will be addressed in this paper: What conditions should the matrix elements $\nu_{\lambda}$ satisfy for the interaction $\hat{V}$ to conserve seniority, either completely or partially? It is important to appreciate that these conditions are weaker than those required for complete solvability on the basis of a dynamical symmetry. To make this point clear, sufficient conditions of solvability associated with a dynamical symmetry are derived in Sect. 2. The conditions for complete seniority conservation are known since long. For completeness, a brief reminder of them is given in Sect. 3 by analyzing the three-particle case. A surprising consequence of these conditions is that they lead to diophantine equations in the spin $j$ of the particles and the component $\lambda$ of the interaction. With the same procedure as in Sect. 3, the four-particle case is analyzed in Sect. 4, revealing the existence of a partial seniority conservation in the $j=9 / 2$ shell. Although this is found to be an exceptional situation for fermions, it occurs frequently for boson systems as is shown in Sect. 5. Applications of the seniority formalism in fermionic systems are presented in Sect. 6. Finally, in Sect. 7 the conclusions of this work are formulated. 


\section{Solvable interactions}

A class of solvable interactions can be found by requiring the existence of a dynamical symmetry which can be viewed as a generalization and refinement of the concept of symmetry $[9,10]$. A dynamical symmetry occurs if the hamiltonian is written in terms of Casimir operators of a set of nested algebras. Its hallmarks are (i) solvability of the complete spectrum, (ii) existence of exact quantum numbers for all eigenstates and (iii) pre-determined structure of the eigenfunctions, independent of the parameters in the hamiltonian.

A general one- plus two-body hamiltonian for a system of identical interacting particles considered here is given by

$$
\hat{H}=\epsilon \hat{N}+\sum_{\lambda} \nu_{\lambda} \hat{V}_{\lambda}
$$

where $\epsilon$ is the single-particle energy. If the hamiltonian can be written as a linear combination of the Casimir operators of the algebras appearing in Eqs. (1) and (2), then the labels $N, v$ and $J$ are good quantum numbers for all eigenstates. In this case the hamiltonian has the form

$$
\hat{H}_{\mathrm{ds}}^{\mathrm{b}}=x_{1} \hat{C}_{1}[\mathrm{U}(n)]+x_{2} \hat{C}_{2}[\mathrm{U}(n)]+x_{3} \hat{C}_{2}[\mathrm{SO}(n)]+x_{4} \hat{C}_{2}[\mathrm{SO}(3)],
$$

or

$$
\hat{H}_{\mathrm{ds}}^{\mathrm{f}}=x_{1} \hat{C}_{1}[\mathrm{U}(n)]+x_{2} \hat{C}_{2}[\mathrm{U}(n)]+x_{3} \hat{C}_{2}[\mathrm{Sp}(n)]+x_{4} \hat{C}_{2}[\mathrm{SU}(2)]
$$

for bosons or fermions, respectively, where the notation $n \equiv 2 j+1$ is used and $\hat{C}_{i}[G]$ denotes the Casimir operator of order $i$ of the algebra $G$. By writing the Casimir operator in terms of $\hat{N}$ and $\hat{V}_{\lambda}$, one obtains a (possibly overcomplete) system of linear equations in the coefficients $\epsilon$ and $\nu_{\lambda}$ of the general hamiltonian. From a simple counting argument it is clear that these equations admit a solution for $j=0,1 / 2,1,3 / 2,2$ and $5 / 2$. For $j>5 / 2$ there are more coefficients than there are Casimir operators. The system of equations then becomes overcomplete, leading to conditions on the coefficients $\nu_{\lambda}$. There will be one condition for $j=3$ or $7 / 2$, two conditions for $j=4$ or $9 / 2$, and so on. The system of equations can be written in general as

$$
\begin{aligned}
& x_{1}=\epsilon-\frac{1}{2 n} \nu_{0}+\frac{n^{4}+n^{3}-41 n^{2}-n+40}{56 n} \nu_{2}-\frac{n^{4}+n^{3}-13 n^{2}-n+12}{56 n} \nu_{4}, \\
& x_{2}=\frac{1}{2 n}\left(\nu_{0}-\frac{n^{3}-41 n+40}{28} \nu_{2}+\frac{n^{3}-13 n+12}{28} \nu_{4}\right),
\end{aligned}
$$




$$
\begin{aligned}
& x_{3}=\frac{1}{2 n}\left(-\nu_{0}+\frac{10}{7} \nu_{2}-\frac{3}{7} \nu_{4}\right), \\
& x_{4}=\frac{\nu_{4}-\nu_{2}}{14}=\frac{\nu_{\lambda}-\nu_{2}}{\lambda(\lambda+1)-6},
\end{aligned}
$$

and

$$
\begin{aligned}
& x_{1}=\epsilon+\frac{1}{2 n} \nu_{0}-\frac{n^{4}-n^{3}-41 n^{2}+n+40}{56 n} \nu_{2}+\frac{n^{4}-n^{3}-13 n^{2}+n+12}{56 n} \nu_{4}, \\
& x_{2}=\frac{1}{2 n}\left(\nu_{0}+\frac{n^{3}-41 n-40}{28} \nu_{2}-\frac{n^{3}-13 n-12}{28} \nu_{4}\right), \\
& x_{3}=\frac{1}{2 n}\left(-\nu_{0}+\frac{10}{7} \nu_{2}-\frac{3}{7} \nu_{4}\right), \\
& x_{4}=\frac{\nu_{4}-\nu_{2}}{14}=\frac{\nu_{\lambda}-\nu_{2}}{\lambda(\lambda+1)-6},
\end{aligned}
$$

for bosons and fermions, respectively. In each case the conditions on the interactions $\nu_{\lambda}$ follow from the last equation which is the same for bosons and fermions,

$$
\nu_{\lambda}=\frac{20-\lambda(\lambda+1)}{14} \nu_{2}-\frac{6-\lambda(\lambda+1)}{14} \nu_{4}, \quad \lambda=6,8,10, \ldots
$$

These are sufficient conditions on the $\nu_{\lambda}$ for the hamiltonian to have a dynamical symmetry, resulting in complete solvability of the spectrum. In the following section the weaker conditions are reviewed which are needed for complete conservation of seniority.

\section{Seniority conservation for three identical particles}

The conditions for complete seniority conservation are known since long for fermions (see, e.g., Refs. [4,5]) and can be derived from the analysis of a system of three particles. This section presents a succinct derivation of the conditions

for seniority conservation for bosons as well as fermions, to prepare the ground for the analysis of a four-particle system, presented in Sect. 4.

\subsection{Conditions for seniority conservation}

Let us recall a few elementary properties of (anti-)symmetric three-particle states [4,5]. A three-particle state can be written as $\left|j^{2}(R) j ; J\right\rangle$ where two particles are first coupled to angular momentum $R$ which is subsequently 
coupled to total angular momentum $J$. This state is not (anti-)symmetric in all three particles; it can be made so by applying the (anti-)symmetry operator $\hat{P}$

$$
\left.\left|j^{3}[I] J\right\rangle \propto \hat{P}\left|j^{2}(I) j ; J\right\rangle=\sum_{R}\left[j^{2}(R) j ; J \mid\right\} j^{3}[I] J\right]\left|j^{2}(R) j ; J\right\rangle,
$$

where $\left.\left[j^{2}(R) j ; J \mid\right\} j^{3}[I] J\right]$ is a three-to-two-particle coefficient of fractional parentage (CFP). The notation in round brackets in $\left|j^{2}(R) j ; J\right\rangle$ implies coupling of two particles to intermediate angular momentum $R$. On the other hand, the square brackets $[I]$ label a three-particle state and indicate that it has been obtained after (anti-)symmetrization of $\left|j^{2}(I) j ; J\right\rangle$. The label $[I]$ defines an overcomplete, non-orthogonal basis, that is, not all $\left|j^{3}[I] J\right\rangle$ states with $I=0,2, \ldots, 2 p$ are independent.

The three-to-two-particle CFP is known in closed form,

$$
\left.\left[j^{2}(R) j ; J \mid\right\} j^{3}[I] J\right]=\frac{1}{\sqrt{N_{j J}^{I}}}\left(\delta_{R I}+2 \sqrt{(2 R+1)(2 I+1)}\left\{\begin{array}{ccc}
j & j & R \\
J & j & I
\end{array}\right\}\right)
$$

with the normalization coefficient

$$
N_{j J}^{I}=3\left(1+2(2 I+1)\left\{\begin{array}{lll}
j & j & I \\
J & j & I
\end{array}\right\}\right)
$$

where the symbol between curly brackets is a Racah coefficient $[4,5]$. Both the overlap matrix and the matrix element of the operator $\hat{V}_{\lambda}$ can be expressed in terms of the CFPs,

$$
\begin{aligned}
\left\langle j^{3}[I] J \mid j^{3}[L] J\right\rangle & \left.\left.=\sum_{R}\left[j^{2}(R) j ; J \mid\right\} j^{3}[I] J\right]\left[j^{2}(R) j ; J \mid\right\} j^{3}[L] J\right], \\
\left\langle j^{3}[I] J\left|\hat{V}_{\lambda}\right| j^{3}[L] J\right\rangle & \left.\left.=3\left[j^{2}(\lambda) j ; J \mid\right\} j^{3}[I] J\right]\left[j^{2}(\lambda) j ; J \mid\right\} j^{3}[L] J\right] .
\end{aligned}
$$

With use of properties of the Racah coefficient the sum over the CFPs in the expression for the overlap matrix can be carried out,

$$
\begin{aligned}
\left\langle j^{3}[I] J \mid j^{3}[L] J\right\rangle & =\frac{3}{\sqrt{N_{j J}^{I} N_{j J}^{L}}}\left(\delta_{I L}+2 \sqrt{(2 I+1)(2 L+1)}\left\{\begin{array}{ccc}
j & j & I \\
J & j & L
\end{array}\right\}\right) \\
& \left.=\frac{3}{\sqrt{N_{j J}^{I}}}\left[j^{2}(I) j ; J \mid\right\} j^{3}[L] J\right],
\end{aligned}
$$


leading to closed expressions for both the overlap matrix and the matrix element of the operator $\hat{V}_{\lambda}$.

\subsection{Diophantine equations for seniority conservation}

Let us begin with the following simpler problem. Can one find the condition for a single component $\hat{V}_{\lambda}$ of the interaction to conserve seniority for a given particle angular momentum $j$ ? Let us first establish a necessary condition for seniority conservation $[4,5]$. By definition the seniority $v=1$ three-particle state is

$$
\left|j^{3}, v=1, J\right\rangle=\left|j^{3}[0] J\right\rangle
$$

where the total angular momentum $J$ must be equal to the angular momentum $j$ of the individual particles. A seniority $v=3$ state originates from a different parent state (i.e., it has $I \neq 0$ ) and is defined to be orthogonal to the seniority $v=1$ state. Hence

$$
\left|j^{3}[I], v=3, J\right\rangle=\left|j^{3}[I] J\right\rangle-\left\langle j^{3}[0] J \mid j^{3}[I] J\right\rangle\left|j^{3}[0] J\right\rangle, \quad I \neq 0 .
$$

Seniority conservation for $\hat{V}_{\lambda}$ requires $\left\langle j^{3}, v=1, J\left|\hat{V}_{\lambda}\right| j^{3}[I], v=3, J\right\rangle=0$ or

$$
\frac{\left\langle j^{3}[0] J\left|\hat{V}_{\lambda}\right| j^{3}[I] J\right\rangle}{\left\langle j^{3}[0] J\left|\hat{V}_{\lambda}\right| j^{3}[0] J\right\rangle}=\left\langle j^{3}[0] J \mid j^{3}[I] J\right\rangle .
$$

With use of the expressions (13) and (14) this condition reduces to

$$
\left.\frac{\left.\left[j^{2}(\lambda) j ; J \mid\right\} j^{3}[I] J\right]}{\left.\left[j^{2}(\lambda) j ; J \mid\right\} j^{3}[0] J\right]}=\frac{3}{\sqrt{N_{j j}^{0}}}\left[j^{2}(0) j ; J \mid\right\} j^{3}[I] J\right] .
$$

From the general expression (10) the following simple cases are obtained:

$$
\begin{aligned}
& \left.\left[j^{2}(0) j ; J \mid\right\} j^{3}[0] J\right]=\sqrt{\frac{2 j+1+2 \sigma}{3(2 j+1)}}, \\
& \left.\left[j^{2}(\lambda) j ; J \mid\right\} j^{3}[0] J\right]=\sigma \sqrt{\frac{4(2 \lambda+1)}{3(2 j+1)(2 j+1+2 \sigma)}}, \quad \lambda \neq 0,
\end{aligned}
$$

where $\sigma \equiv(-)^{2 j}$ is +1 for bosons and -1 for fermions. This leads to the following condition (for $\lambda \neq 0$ ) valid for bosons and fermions: 


$$
\delta_{\lambda I}+2 \sqrt{(2 \lambda+1)(2 I+1)}\left\{\begin{array}{ccc}
j & j \lambda \\
j & j I
\end{array}\right\}=\frac{4 \sqrt{(2 \lambda+1)(2 I+1)}}{(2 j+1)(2 j+1+2 \sigma)} .
$$

For seniority to be conserved by the interaction $\hat{V}_{\lambda}$, this equation must be satisfied for all even intermediate angular momenta $2 \leq I \leq 2 p$. Let us take $I=2$ and first consider $\lambda \neq I$. For bosons the condition (20) then leads to the equation

$$
\begin{aligned}
3 \lambda^{4}+6 \lambda^{3}-6[2 j(j+1)-1] \lambda^{2} & -3[4 j(j+1)-1] \lambda \\
& +2 j(j+1)(2 j-1)(2 j+1)=0 .
\end{aligned}
$$

This should be considered as a diophantine equation in $\lambda$ since only (positive, even) integer solutions in $\lambda$ have a physical meaning. For $j=2$ the diophantine equation (21) is satisfied for $\lambda=4$. This confirms a known result namely that any interaction between $d$ bosons is integrable and conserves seniority, hence also $\hat{V}_{4}$. More surprisingly, the equation is also satisfied for $j=5$ and $\lambda=4$ and one may verify that in that case the condition (20) is equally valid for $I=4$, 6, 8 and 10. This means that a $\hat{V}_{4}$ interaction between $h$ bosons conserves seniority. The result is illustrated in Fig. 1 where the $J=2$ spectrum of six $h$ bosons is shown as a function of the interaction strength $\nu_{4}$. To split states with different seniorities, a constant (repulsive) pairing interaction $\hat{V}_{0}$ is taken to which a variable $\hat{V}_{4}$ part is added. The resulting hamiltonian is diagonalized numerically with the code ArbModel [11] which can compute the properties of a system consisting of an arbitrary combination of bosons and/or fermions interacting through two-body forces. The figure confirms that there are no avoided crossings for $h$ bosons since the only crossings that do occur are between levels of different seniority and those are unavoided. For comparison, the $J=2$ spectrum of six $g$ bosons is also shown as a function of the strength $\nu_{4}$, and in this case the crossings are avoided.

To complete the analysis of the boson case, for $\lambda=I=2$ the condition (20) leads to

$$
8 j^{5}+60 j^{4}+50 j^{3}-375 j^{2}-373 j+630=0 .
$$

This equation has the integer solutions $j=1$ and $j=2$ as should be since any interaction between $p$ or $d$ bosons is integrable and conserves seniority.

For fermions the condition (20) leads to the diophantine equation

$$
\begin{aligned}
3 \lambda^{4}+6 \lambda^{3}-6[2 j(j+1)-1] \lambda^{2} & -3[4 j(j+1)-1] \lambda \\
+ & 2 j(j+1)(2 j+1)(2 j+3)=0 .
\end{aligned}
$$




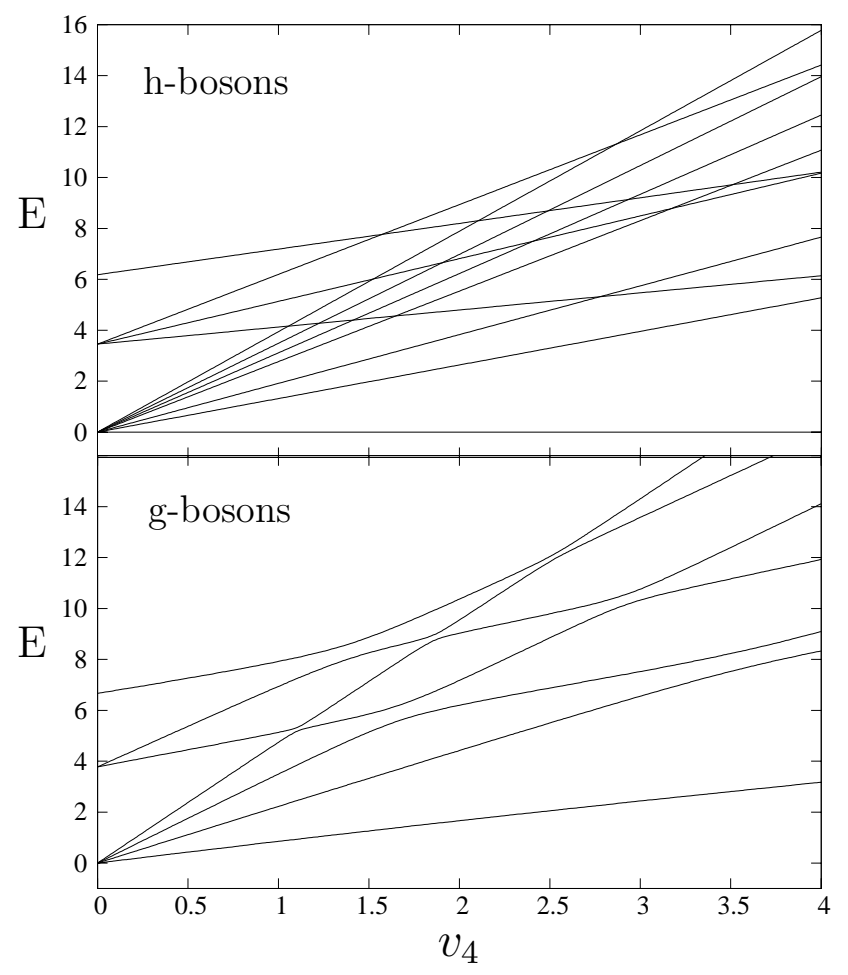

Fig. 1. The energy spectrum of six $h$ bosons (top) or six $g$ bosons (bottom) coupled to total angular momentum $J=2$ as a function of the interaction strength $\nu_{4}$. For the $h$ bosons all crossings are unavoided which is a consequence of the conservation of seniority. In contrast, for $g$ bosons crossings are avoided since there is no conservation of seniority.

This equation is satisfied for $(j, \lambda)=(5 / 2,4),(7 / 2,4)$ and $(7 / 2,6)$ which confirms the known result that for fermions with $j \leq 7 / 2$ any interaction is diagonal in seniority $[4,5]$. Finally, to complete the analysis for fermions, for $\lambda=I=2$ the condition (20) leads to

$$
8 j^{5}-20 j^{4}-110 j^{3}+245 j^{2}+327 j-630=0
$$

which has the half-odd-integer solutions $j=3 / 2,5 / 2$ and $7 / 2$, again as should be.

As an amusing aside, note that the diophantine equations (22) and (24) also allow negative solutions, namely, $-5 / 2,-7 / 2$ and $-9 / 2$ for the bosons and -2 and -3 for the fermions. It thus transpires that the negative solutions $x$ for the particles of one statistics correspond to the positive solutions $-x-1$ for the particles of the other statistics. 


\subsection{Seniority conservation for a general interaction}

Let us next consider the condition of seniority conservation for a general interaction $\hat{V}=\sum_{\lambda} \nu_{\lambda} \hat{V}_{\lambda}$. The analogue of the condition (17) is

$$
\frac{\left\langle j^{3}[0] J|\hat{V}| j^{3}[I] J\right\rangle}{\left\langle j^{3}[0] J|\hat{V}| j^{3}[0] J\right\rangle}=\left\langle j^{3}[0] J \mid j^{3}[I] J\right\rangle
$$

where it is again assumed that $J=j$ and $I \neq 0$. This leads to the following relation between the coefficients $\nu_{\lambda}$ :

$$
\begin{aligned}
&\left.\sum_{\lambda}\left[j^{2}(\lambda) j ; J \mid\right\} j^{3}[0] J\right]\left.\left(\left[j^{2}(0) j ; J \mid\right\} j^{3}[0] J\right]\left[j^{2}(\lambda) j ; J \mid\right\} j^{3}[I] J\right] \\
&\left.\left.\left.-\left[j^{2}(0) j ; J \mid\right\} j^{3}[I] J\right]\left[j^{2}(\lambda) j ; J \mid\right\} j^{3}[0] J\right]\right) \nu_{\lambda}=0 .
\end{aligned}
$$

With use of the explicit expressions for the various CFPs this can be cast into the following form:

$$
\begin{array}{r}
\sum_{\lambda=2}^{2 p} \sqrt{2 \lambda+1}\left(\delta_{\lambda I}+2 \sqrt{(2 \lambda+1)(2 I+1)}\left\{\begin{array}{ll}
j & j \\
j & j
\end{array}\right\}\right. \\
\left.-\frac{4 \sqrt{(2 \lambda+1)(2 I+1)}}{(2 j+1)(2 j+1+2 \sigma)}\right) \nu_{\lambda}=0 .
\end{array}
$$

This condition has been derived previously in a variety of ways mostly for fermions $[4,5,12,13]$. The result $(27)$ shows that a simple expression exists which covers both the boson and the fermion case. Although Eq. (27) determines all constraints on the matrix elements $\nu_{\lambda}$ by varying $I$ between 2 and $2 p$, it does not tell us how many of those are independent. This number turns out to be $\lfloor j / 3\rfloor$ for bosons and $\lfloor(2 j-3) / 6\rfloor$ for fermions, the number of independent seniority $v=3$ states [14]. Hence no condition on the matrix elements $\nu_{\lambda}$ follows for $j=1$ and 2 , and for $j=3 / 2,5 / 2$ and $7 / 2$. For higher values of $j$ one finds

$$
\begin{aligned}
j=3: & 11 \nu_{2}-18 \nu_{4}+7 \nu_{6}=0 \\
j=4: & : 65 \nu_{2}-30 \nu_{4}-91 \nu_{6}+56 \nu_{8}=0 \\
j=5: & 3230 \nu_{2}-2717 \nu_{6}-3978 \nu_{8}+3465 \nu_{10}=0 \\
j=6: & 22610 \nu_{2}+4788 \nu_{4}-8099 \nu_{6}-24106 \nu_{8}-23793 \nu_{10}+28600 \nu_{12}=0, \\
& 90440 \nu_{2}+156807 \nu_{4}-409136 \nu_{6}+290666 \nu_{8}-275352 \nu_{10} \\
& +146575 \nu_{12}=0,
\end{aligned}
$$


and

$$
\begin{array}{ccc}
j=9 / 2: & 65 \nu_{2}-315 \nu_{4}+403 \nu_{6}-153 \nu_{8}=0 \\
j=11 / 2: & 1020 \nu_{2}-3519 \nu_{4}+637 \nu_{6}+4403 \nu_{8}-2541 \nu_{10}=0 \\
j=13 / 2: & 1615 \nu_{2}-4275 \nu_{4}-1456 \nu_{6}+3196 \nu_{8}+5145 \nu_{10}-4225 \nu_{12}=0 . \\
j=15 / 2: & 1330 \nu_{2}-2835 \nu_{4}-1807 \nu_{6}+612 \nu_{8}+3150 \nu_{10}+3175 \nu_{12} \\
& -3625 \nu_{14}=0 \\
& 77805 \nu_{2}-169470 \nu_{4}-85527 \nu_{6}-4743 \nu_{8}+222768 \nu_{10} \\
& +168025 \nu_{12}-208858 \nu_{14}=0 .
\end{array}
$$

for bosons and fermions, respectively. The coefficient $\nu_{0}$ is absent from all equations since pairing $(\lambda=0)$ is known to conserve seniority. Note that for $j=5$ there is no term in $\nu_{4}$ which is consistent with the results of Sect. 3.2.

These results establish the necessary conditions for an interaction to be seniority conserving by imposing vanishing matrix elements between seniority $v=1$ and $v=3$ three-particle states. It can be shown with use of generic properties of CFPs that these are also sufficient conditions [4,5]. This means that the conditions (27) are necessary and sufficient for seniority to be a conserved quantum number in a system of $N$ identical particles.

\section{Partial seniority conservation for four identical particles}

Let us now turn our attention to the four-particle case. This analysis will, of course, confirm the results of the previous section pertaining to seniority conservation in $N$-particle systems but the particular interest of this section concerns the possibility of partial seniority conservation. It is important to clarify first what is meant by partial dynamical symmetry which is an enlargement of the concept of dynamical symmetry as defined in Sect. 2. The idea is to relax the conditions of complete solvability and this can be done in essentially two different ways:

(1) Some of the eigenstates keep all of the quantum numbers. In this case the properties of solvability, good quantum numbers, and symmetrydictated structure are fulfilled exactly, but only by a subset of eigenstates $[15,16,17]$.

(2) All eigenstates keep some of the quantum numbers. In this case eigenstates are not solvable, yet some quantum numbers (of the conserved 
symmetries) are retained. In general, this type of partial dynamical symmetry arises if the hamiltonian preserves some of the quantum numbers in a dynamical-symmetry classification while breaking others [18,19].

Combinations of (1) and (2) are possible as well, for example, if some of the eigenstates keep some of the quantum numbers [20].

A further clarification concerning the notion of solvability is needed. One might argue, for example, that, as long as the hamiltonian matrix is of finite size (as it is always the case in this paper), its eigenvalues and eigenvectors can be determined in a finite number of steps and that as a consequence the secular equation is exactly solvable. The condition of solvability adopted here is stronger, and requires the property of a predetermined structure of the eigenvector, independent of the parameters in the hamiltonian. So, an eigenstate will be called solvable only if its structure is independent of the interaction matrix elements $\nu_{\lambda}$. This is also the definition adopted by Talmi [21] who showed that, if an eigenstate is solvable in this sense, its energy is a linear combination of the $\nu_{\lambda}$ with coefficients that are rational non-negative numbers.

How do seniority-conserving interactions fit in this classification? If the conditions (27) are satisfied by an interaction $\hat{V}$, all its eigenstates carry the seniority quantum number $v$ and, consequently, the second type of partial dynamical symmetry applies. In addition, some of the eigenstates are completely solvable. For example, the eigenstate with seniority $v=0$ of a seniority-conserving interaction (this corresponds to the ground state of an even-even nucleus) has a structure independent of the hamiltonian's parameters and an analytic expression is available for its energy. So, one concludes that seniority-conserving interactions in general satisfy the second type of partial dynamical symmetry but with the added feature that some states are completely solvable.

And what about more general interactions? More specifically, is it possible to construct seniority-mixing interactions, some of the eigenstates of which have good seniority? An example was given by Escuderos and Zamick [22] who pointed out that four fermions in a $j=9 / 2$ shell display one $J=4$ and one $J=6$ state both of which have seniority $v=4$ for an arbitrary interaction. This is an example of a partial symmetry, where seniority is broken for most but not for all states.

\subsection{Conditions for seniority conservation}

To shed light on the problem of partial seniority conservation, the four-particle case can be analyzed, displaying a close analogy with the three-particle case

reviewed in Sect. 3. A four-particle state can be written as $\left|j^{2}(R) j^{2}\left(R^{\prime}\right) ; J\right\rangle$ where two particles are first coupled to angular momentum $R$, the next two 
particles to $R^{\prime}$ and the intermediate angular momenta $R$ and $R^{\prime}$ to total $J$. This state is not (anti-)symmetric in all four particles and can be made so by applying the (anti-)symmetry operator $\hat{P}$,

$$
\begin{aligned}
\left|j^{4}\left[I I^{\prime}\right] J\right\rangle & \propto \hat{P}\left|j^{2}(I) j^{2}\left(I^{\prime}\right) ; J\right\rangle \\
& \left.=\sum_{R R^{\prime}}\left[j^{2}(R) j^{2}\left(R^{\prime}\right) ; J \mid\right\} j^{4}\left[I I^{\prime}\right] J\right]\left|j^{2}(R) j^{2}\left(R^{\prime}\right) ; J\right\rangle
\end{aligned}
$$

where $\left.\left[j^{2}(R) j^{2}\left(R^{\prime}\right) ; J \mid\right\} j^{4}\left[I I^{\prime}\right] J\right]$ is a four-to-two-particle CFP. The notation in square brackets $\left[I I^{\prime}\right]$ implies that the state $(28)$ is constructed from a parent with intermediate angular momenta $I$ and $I^{\prime}$. It is implicitly assumed that $I$ and $I^{\prime}$ as well as $R$ and $R^{\prime}$ are even.

The remarks made in the three-particle case concerning non-orthogonality and over-completeness apply also here. Because of the difficulties associated with a non-orthogonal basis, it will sometimes be advantageous to convert to an orthogonal one, which can be achieved through a standard Gram-Schmidt procedure. Given an ordered set of $p$ non-orthogonal bases states,

$$
\left|j^{4}\left[I_{1} I_{1}^{\prime}\right] J\right\rangle,\left|j^{4}\left[I_{2} I_{2}^{\prime}\right] J\right\rangle, \ldots,\left|j^{4}\left[I_{p} I_{p}^{\prime}\right] J\right\rangle
$$

the orthonormalized bases states will be denoted as

$$
\begin{aligned}
\left|j^{4}\left[\widetilde{I_{1} I_{1}^{\prime}}\right] J\right\rangle & =\frac{1}{\sqrt{o_{11}}}\left|j^{4}\left[I_{1} I_{1}^{\prime}\right] J\right\rangle \\
\left|j^{4}\left[\widetilde{I_{2} I_{2}^{\prime}}\right] J\right\rangle & =\frac{1}{\sqrt{o_{22}-\left(\tilde{o}_{21}\right)^{2}}}\left(\left|j^{4}\left[I_{2} I_{2}^{\prime}\right] J\right\rangle-\tilde{o}_{21}\left|j^{4}\left[\widetilde{I_{1} I_{1}^{\prime}}\right] J\right\rangle\right) \\
& \vdots \\
\left|j^{4}\left[\widetilde{I_{k} I_{k}^{\prime}}\right] J\right\rangle= & \frac{1}{\sqrt{N_{k}}}\left(\left|j^{4}\left[I_{k} I_{k}^{\prime}\right] J\right\rangle-\sum_{i=1}^{k-1} \tilde{o}_{k i}\left|j^{4}\left[\widetilde{I_{i} I_{i}^{\prime}}\right] J\right\rangle\right) \\
& \vdots
\end{aligned}
$$

until $k=p$, with

$$
N_{k}=o_{k k}-\sum_{i=1}^{k-1}\left(\tilde{o}_{k i}\right)^{2}, \quad \tilde{o}_{k i}=\left\langle j^{4}\left[I_{k} I_{k}^{\prime}\right] J \mid j^{4}\left[\widetilde{I_{i} I_{i}^{\prime}}\right] J\right\rangle .
$$

The four-to-two-particle CFP is known in closed form,

$$
\left.\left[j^{2}(R) j^{2}\left(R^{\prime}\right) ; J \mid\right\} j^{4}\left[I I^{\prime}\right] J\right]
$$




$$
=\frac{1}{\sqrt{N_{j J}^{I I^{\prime}}}}\left(\delta_{R I} \delta_{R^{\prime} I^{\prime}}+(-)^{J} \delta_{R I^{\prime}} \delta_{R^{\prime} I}+4 \sigma\left[\begin{array}{ccc}
j & j & R \\
j & j & R^{\prime} \\
I & I^{\prime} & J
\end{array}\right]\right),
$$

where the symbol in square brackets is related to the $9 j$ symbol through

$$
\left[\begin{array}{ccc}
j_{1} & j_{2} & J_{12} \\
j_{3} & j_{4} & J_{34} \\
J_{13} & J_{24} & J
\end{array}\right]=\hat{J}_{12} \hat{J}_{34} \hat{J}_{13} \hat{J}_{24}\left\{\begin{array}{ccc}
j_{1} & j_{2} & J_{12} \\
j_{3} & j_{4} & J_{34} \\
J_{13} & J_{24} & J
\end{array}\right\},
$$

with $\hat{J}=\sqrt{2 J+1}$. With use of the following sum over even values of $R$ and $R^{\prime}$,

$$
\begin{aligned}
\sum_{R R^{\prime} \text { even }}\left[\begin{array}{ccc}
j & j & R \\
j & j & R^{\prime} \\
I & I^{\prime} & J
\end{array}\right] & {\left[\begin{array}{ccc}
j & j & R \\
j & j & R^{\prime} \\
L & L^{\prime} & J
\end{array}\right]=\frac{1}{4}\left(\delta_{I L} \delta_{I^{\prime} L^{\prime}}+(-)^{I+I^{\prime}+J} \delta_{I L^{\prime}} \delta_{I^{\prime} L}\right.} \\
& \left.+2 \sigma\left((-)^{I+L}+(-)^{I^{\prime}+L^{\prime}}\right)\left[\begin{array}{ccc}
j & j & I \\
j & j & I^{\prime} \\
L & L^{\prime} & J
\end{array}\right]\right)
\end{aligned}
$$

the normalization coefficient can be obtained as

$$
N_{j J}^{I I^{\prime}}=6\left(1+(-)^{J} \delta_{I I^{\prime}}+4 \sigma\left[\begin{array}{ccc}
j & j & I \\
j & j & I^{\prime} \\
I & I^{\prime} & J
\end{array}\right]\right) .
$$

Both the overlap matrix and the matrix element of the operator $\hat{V}_{\lambda}$ can be expressed in terms of the CFPs,

$$
\begin{aligned}
& \left\langle j^{4}\left[I I^{\prime}\right] J \mid j^{4}\left[L L^{\prime}\right] J\right\rangle \\
& \left.\left.\quad=\sum_{R R^{\prime}}\left[j^{2}(R) j^{2}\left(R^{\prime}\right) ; J \mid\right\} j^{4}\left[I I^{\prime}\right] J\right]\left[j^{2}(R) j^{2}\left(R^{\prime}\right) ; J \mid\right\} j^{4}\left[L L^{\prime}\right] J\right], \\
& \left\langle j^{4}\left[I I^{\prime}\right] J\left|\hat{V}_{\lambda}\right| j^{4}\left[L L^{\prime}\right] J\right\rangle
\end{aligned}
$$




$$
\left.\left.=6 \sum_{R}\left[j^{2}(R) j^{2}(\lambda) ; J \mid\right\} j^{4}\left[I I^{\prime}\right] J\right]\left[j^{2}(R) j^{2}(\lambda) ; J \mid\right\} j^{4}\left[L L^{\prime}\right] J\right] .
$$

With use of the result (34) the first of these sums can be carried out, yielding the expression

$$
\begin{aligned}
& \left\langle j^{4}\left[I I^{\prime}\right] J \mid j^{4}\left[L L^{\prime}\right] J\right\rangle \\
& \quad=\frac{6}{\sqrt{N_{j J}^{I I^{\prime}} N_{j J}^{L L^{\prime}}}}\left(\delta_{I L} \delta_{I^{\prime} L^{\prime}}+(-)^{J} \delta_{I L^{\prime}} \delta_{I^{\prime} L}+4 \sigma\left[\begin{array}{ccc}
j & j & I \\
j & j & I^{\prime} \\
L & L^{\prime} & J
\end{array}\right]\right) \\
& \left.=\frac{6}{\sqrt{N_{j J}^{I I^{\prime}}}}\left[j^{2}(I) j^{2}\left(I^{\prime}\right) ; J \mid\right\} j^{4}\left[L L^{\prime}\right] J\right] .
\end{aligned}
$$

The four-particle case with $J=0$ is equivalent to three particles coupled to $J=j$ which was considered in Sect. 3. Therefore it is assumed in the following that $J \neq 0$, corresponding to four-particle states with seniority $v=2$ or $v=4$. By definition the seniority $v=2$ four-particle state is

$$
\left|j^{4}, v=2, J\right\rangle=\left|j^{4}[0 J] J\right\rangle
$$

A seniority $v=4$ state is orthogonal to this state and can thus be written as

$$
\left|j^{4}\left[I I^{\prime}\right], v=4, J\right\rangle=\left|j^{4}\left[I I^{\prime}\right] J\right\rangle-\left\langle j^{4}\left[I I^{\prime}\right] J \mid j^{4}[0 J] J\right\rangle\left|j^{4}[0 J] J\right\rangle .
$$

There can be more than one seniority $v=4$ state for a given $J$ in which case the indices $\left[I I^{\prime}\right]$ may serve as an additional label. Seniority conservation of $\hat{V}_{\lambda}$ implies $\left\langle j^{4}[0 J] J\left|\hat{V}_{\lambda}\right| j^{4}\left[I I^{\prime}\right], v=4, J\right\rangle=0$ or

$$
\frac{\left\langle j^{4}[0 J] J\left|\hat{V}_{\lambda}\right| j^{4}\left[I I^{\prime}\right] J\right\rangle}{\left\langle j^{4}[0 J] J\left|\hat{V}_{\lambda}\right| j^{4}[0 J] J\right\rangle}=\left\langle j^{4}[0 J] J \mid j^{4}\left[I I^{\prime}\right] J\right\rangle .
$$

With use of the expressions (37) and (38) this condition reduces to

$$
\begin{aligned}
& \frac{\left.\left.\sum_{R}\left[j^{2}(R) j^{2}(\lambda) ; J \mid\right\} j^{4}\left[I I^{\prime}\right] J\right]\left[j^{2}(R) j^{2}(\lambda) ; J \mid\right\} j^{4}[0 J] J\right]}{\left.\left.\sum_{R}\left[j^{2}(R) j^{2}(\lambda) ; J \mid\right\} j^{4}[0 J] J\right]\left[j^{2}(R) j^{2}(\lambda) ; J \mid\right\} j^{4}[0 J] J\right]} \\
& \left.=\frac{6}{\sqrt{N_{j J}^{0 J}}}\left[j^{2}(0) j^{2}(J) ; J \mid\right\} j^{4}\left[I I^{\prime}\right] J\right] .
\end{aligned}
$$


In the same way as in the three-particle case one can also derive the condition of seniority conservation for a general interaction $\hat{V}=\sum_{\lambda} \nu_{\lambda} \hat{V}_{\lambda}$. The condition

$$
\frac{\left\langle j^{4}[0 J] J|\hat{V}| j^{4}\left[I I^{\prime}\right] J\right\rangle}{\left\langle j^{4}[0 J] J|\hat{V}| j^{4}[0 J] J\right\rangle}=\left\langle j^{4}[0 J] J \mid j^{4}\left[I I^{\prime}\right] J\right\rangle,
$$

leads to the following equation:

$$
\begin{aligned}
& \left.\sum_{R \lambda}\left[j^{2}(R) j^{2}(\lambda) ; J \mid\right\} j^{4}[0 J] J\right] \\
& \left.\quad \times\left(\left[j^{2}(J) j^{2}(0) ; J \mid\right\} j^{4}[0 J] J\right]\left[j^{2}(R) j^{2}(\lambda) ; J \mid\right\} j^{4}\left[I I^{\prime}\right] J\right] \\
& \left.\left.\left.\quad-\left[j^{2}(J) j^{2}(0) ; J \mid\right\} j^{4}\left[I I^{\prime}\right] J\right]\left[j^{2}(R) j^{2}(\lambda) ; J \mid\right\} j^{4}[0 J] J\right]\right) \nu_{\lambda}=0 .
\end{aligned}
$$

Note the formal equivalence of this condition to the one obtained in the threeparticle case, Eq. (26). Insertion of the values for the four-to-two-particle CFPs yields exactly the same constraints as those derived in the three-particle case.

\subsection{Partial seniority conservation}

Let us now turn our attention to the problem of partial seniority conservation and derive the conditions for an interaction $\hat{V}$ to have some four-particle eigenstates with good seniority. Note that there are a number of 'trivial' examples of this. For example, if the total angular momentum $J$ is odd, a four-particle state cannot be of seniority $v=0$ or $v=2$ and must necessarily have seniority $v=4$. Also, for $J>2 p$ the four-particle state must be of seniority $v=4$. These trivial cases are not of interest here but rather the situation where both $v=2$ and $v=4$ occur for the same $J$ and where a general interaction $\hat{V}$ mixes the $v=2$ state with a subset of the $v=4$ states but not with all. Let us denote such a special $v=4$ state as $\mid j^{4}, v=4$, s, $\left.J\right\rangle$ and expand it in terms of the basis $\left|j^{4}\left[I I^{\prime}\right] J\right\rangle$ discussed previously,

$$
\left|j^{4}, v=4, \mathrm{~s}, J\right\rangle=\sum_{I I^{\prime} \in \wp} \eta_{I I^{\prime}}\left|j^{4}\left[I I^{\prime}\right] J\right\rangle
$$

where the sum runs over $q$ linearly independent combinations $\left[I I^{\prime}\right]$ in the set $\wp$, as many as there are independent four-particle states with angular momentum

$J$. For this state to be an eigenstate of $\hat{V}$ it should satisfy

$$
\hat{V}\left|j^{4}, v=4, \mathrm{~s}, J\right\rangle=E\left|j^{4}, v=4, \mathrm{~s}, J\right\rangle,
$$


in addition to the condition of orthogonality to the $v=2$ state,

$$
\left\langle j^{4}[0 J] J \mid j^{4}, v=4, \mathrm{~s}, J\right\rangle=0 .
$$

Let us now focus on bosons with $3 \leq j \leq 5$ or fermions with $9 / 2 \leq j \leq 13 / 2$. In these cases a general interaction can be written as a single component $\hat{V}_{\lambda}$ plus an interaction $\hat{V}^{\prime}$ that conserves seniority. The conditions (46) and (47) must therefore be checked for a single $\lambda$ component only, which can be arbitrarily chosen. Hence one arrives at the conditions

$$
\sum_{I I^{\prime} \in \wp} \eta_{I I^{\prime}}\left\langle j^{4}\left[L L^{\prime}\right] J\left|\hat{V}_{\lambda}\right| j^{4}\left[I I^{\prime}\right] J\right\rangle=E_{\lambda} \sum_{I I^{\prime} \in \wp} \eta_{I I^{\prime}}\left\langle j^{4}\left[L L^{\prime}\right] J \mid j^{4}\left[I I^{\prime}\right] J\right\rangle
$$

for the different indices $\left[L L^{\prime}\right]$ in the set $\wp$, and

$$
\sum_{I I^{\prime} \in \wp} \eta_{I I^{\prime}}\left\langle j^{4}[0 J] J\left|\hat{V}_{\lambda}\right| j^{4}\left[I I^{\prime}\right] J\right\rangle=0 .
$$

There are $q+1$ unknowns: the $q$ coefficients $\eta_{I I^{\prime}}$ and the energy $E_{\lambda}$. Equations (48) and (49) are also $q+1$ in number and, together with the appropriate normalization condition for the $\eta_{I I^{\prime}}$, they define an overcomplete set of equations in $\left\{\eta_{I I^{\prime}}, E_{\lambda}\right\}$, not satisfied in general but possibly for special values of $j$ and $J$. Furthermore, according to the preceding discussion, if these equations are satisfied for one $\lambda$, they must be valid for all $\lambda$ and in each case the solution yields $E_{\lambda}$, the eigenvalue of $\hat{V}_{\lambda}$.

A symbolic solution of Eqs. (48) and (49) (for general $j$ and $J$ ) is difficult to obtain but, using the expressions derived previously for the various matrix elements, it is straightforward to find a particular solution for given $j$ and $J$. In this way the finding of Refs. [22,23] is confirmed, that is, Eqs. (48) and (49) have a solution for $j=9 / 2, J=4$ and for $j=9 / 2, J=6$. The resulting solvable states are given by

$$
\begin{aligned}
& \left|(9 / 2)^{4}, v=4, \mathrm{~s}, J=4\right\rangle=\sqrt{\frac{25500}{25591}}\left|(9 / 2)^{4}[\widetilde{22}] 4\right\rangle-\sqrt{\frac{91}{25591}}\left|(9 / 2)^{4}[\widetilde{24}] 4\right\rangle, \\
& \left|(9 / 2)^{4}, v=4, \mathrm{~s}, J=6\right\rangle=\sqrt{\frac{27132}{27257}}\left|(9 / 2)^{4}[\widetilde{24}] 6\right\rangle+\sqrt{\frac{125}{27257}}\left|(9 / 2)^{4}[\widetilde{26}] 6\right\rangle .
\end{aligned}
$$

These states are identical to those of Eq. (8) of Ref. [24] but written here in the Gram-Schmidt basis defined in Eq. (30). For the definition of this basis one starts, for $J=4$, from the non-orthogonal set $\left|(9 / 2)^{4}\left[I_{k} I_{k}^{\prime}\right] 4\right\rangle$ with $\left[I_{k} I_{k}^{\prime}\right]=$ [04], [22] and [24]. The second and third states obtained after the GramSchmidt orthonormalization are orthogonal to $\left|(9 / 2)^{4}[04] 4\right\rangle$, and hence have by 
definition seniority $v=4$. A similar argument is valid for $J=6$ where the nonorthogonal set $\left|(9 / 2)^{4}\left[I_{k} I_{k}^{\prime}\right] 6\right\rangle$ has $\left[I_{k} I_{k}^{\prime}\right]=[06],[24]$ and [26]. Consequently, the states (50) have seniority $v=4$.

Furthermore, for each choice of $\lambda$, the solution of the Eqs. (48) and (49) yields $E_{\lambda}$ and these can be used to derive the following energy expressions:

$$
\begin{aligned}
& E\left[(9 / 2)^{4}, v=4, \mathrm{~s}, J=4\right]=\frac{68}{33} \nu_{2}+\nu_{4}+\frac{13}{15} \nu_{6}+\frac{114}{55} \nu_{8}, \\
& E\left[(9 / 2)^{4}, v=4, \mathrm{~s}, J=6\right]=\frac{19}{11} \nu_{2}+\frac{12}{13} \nu_{4}+\nu_{6}+\frac{336}{143} \nu_{8} .
\end{aligned}
$$

The wave functions of the two states are pre-determined and their energies are linear combinations of the $\nu_{\lambda}$ with coefficients that are rational non-negative numbers. These results are valid for an arbitrary interaction among $j=9 / 2$ fermions. According to the discussion of Ref. [21], the states are solvable, independent of whether the interaction conserves seniority or not.

These results are, in fact, rather surprising as can be seen from the structure of the energy matrices for a general interaction $\hat{V}$. For the $(9 / 2)^{4}$ states with angular momentum $J=4$ one finds

$$
\left[\begin{array}{ccc}
E\left[(9 / 2)^{4}[04] 4\right] & \frac{1}{495} \sqrt{\frac{14}{2119}} \Delta E_{1} & \frac{2}{429} \sqrt{\frac{170}{489}} \Delta E_{1} \\
\frac{1}{495} \sqrt{\frac{14}{2119}} \Delta E_{1} & E\left[(9 / 2)^{4}[22] 4\right] & \frac{10}{5379} \sqrt{\frac{595}{39}} \Delta E_{2} \\
\frac{2}{429} \sqrt{\frac{170}{489}} \Delta E_{1} & \frac{10}{5379} \sqrt{\frac{595}{39}} \Delta E_{2} & E\left[(9 / 2)^{4}[24] 4\right]
\end{array}\right]
$$

where the diagonal elements are given by

$$
\begin{aligned}
& E\left[(9 / 2)^{4}[04] 4\right]=\frac{3}{5} \nu_{0}+\frac{67}{99} \nu_{2}+\frac{746}{715} \nu_{4}+\frac{1186}{495} \nu_{6}+\frac{918}{715} \nu_{8}, \\
& E\left[(9 / 2)^{4}[22] 4\right]=\frac{33161}{16137} \nu_{2}+\frac{1800}{1793} \nu_{4}+\frac{70382}{80685} \nu_{6}+\frac{18547}{8965} \nu_{8}, \\
& E\left[(9 / 2)^{4}[24] 4\right]=\frac{2584}{5379} \nu_{2}+\frac{48809}{23309} \nu_{4}+\frac{65809}{26895} \nu_{6}+\frac{114066}{116545} \nu_{8},
\end{aligned}
$$

while in the off-diagonal elements the following combinations of interaction matrix elements $\nu_{J}$ occur:

$$
\begin{aligned}
& \Delta E_{1}=-65 \nu_{2}+315 \nu_{4}-403 \nu_{6}+153 \nu_{8} \\
& \Delta E_{2}=-13 \nu_{2}+9 \nu_{4}+13 \nu_{6}-9 \nu_{8} .
\end{aligned}
$$


The basis which is used for constructing the energy matrix (52) are the states $\left.\left|(9 / 2)^{4}\left[I_{k} I_{k}^{\prime}\right] 4\right\rangle\right\rangle$ discussed above. If the combination $\Delta E_{1}$ vanishes in the matrix (52), no mixing occurs between the seniority $v=2$ and $v=4$ states, and seniority is a good quantum number for the three eigenstates, in agreement with the discussion of Subsect. 3.3.

For completeness, the corresponding expressions for $J=6$ are

$$
\left[\begin{array}{ccc}
E\left[(9 / 2)^{4}[06] 6\right] & -\frac{1}{1287} \sqrt{\frac{5}{97}} \Delta E_{1} & \frac{2}{2145} \sqrt{\frac{2261}{291}} \Delta E_{1} \\
-\frac{1}{1287} \sqrt{\frac{5}{97}} \Delta E_{1} & E\left[(9 / 2)^{4}[24] 6\right] & \frac{10}{5379} \sqrt{\frac{595}{39}} \Delta E_{2} \\
\frac{2}{2145} \sqrt{\frac{2261}{291}} \Delta E_{1} & \frac{10}{5379} \sqrt{\frac{595}{39}} \Delta E_{2} & E\left[(9 / 2)^{4}[26] 6\right]
\end{array}\right]
$$

with the diagonal elements

$$
\begin{aligned}
& E\left[(9 / 2)^{4}[06] 6\right]=\frac{3}{5} \nu_{0}+\frac{34}{99} \nu_{2}+\frac{1186}{715} \nu_{4}+\frac{658}{495} \nu_{6}+\frac{1479}{715} \nu_{8}, \\
& E\left[(9 / 2)^{4}[24] 6\right]=\frac{33049}{19206} \nu_{2}+\frac{25733}{27742} \nu_{4}+\frac{19331}{19206} \nu_{6}+\frac{65059}{27742} \nu_{8}, \\
& E\left[(9 / 2)^{4}[26] 6\right]=\frac{1007}{3201} \nu_{2}+\frac{26370}{13871} \nu_{4}+\frac{7723}{3201} \nu_{6}+\frac{19026}{13871} \nu_{8},
\end{aligned}
$$

while the same combinations (54) occur in the off-diagonal elements.

The energy matrices (52) and (55) are $3 \times 3$ and, generally, none of the offdiagonal elements vanishes. The eigenvalues therefore are roots of a cubic equation and one may expect them to be complicated algebraic expressions in terms of the interaction matrix elements $\nu_{J}$. Surprisingly, this is not the case and, for each of the matrices, one eigenenergy is particularly simple and given by one of the expressions in Eq. (51).

From Eq. (51) the following difference between the excitation energies is derived:

$$
\begin{gathered}
E_{\mathrm{x}}\left[(9 / 2)^{4}, v=4, \mathrm{~s}, J=6\right]-E_{\mathrm{x}}\left[(9 / 2)^{4}, v=4, \mathrm{~s}, J=4\right] \\
=-\frac{1}{3} \nu_{2}-\frac{1}{13} \nu_{4}+\frac{2}{15} \nu_{6}+\frac{18}{65} \nu_{8} .
\end{gathered}
$$

Since the sum of the coefficients of the matrix elements in the expression (57) is zero, one can make the replacement $\nu_{\lambda} \rightarrow \nu_{\lambda}-\nu_{0}$ to arrive at the result

$$
E_{\mathrm{x}}\left[(9 / 2)^{4}, v=4, \mathrm{~s}, J=6\right]-E_{\mathrm{x}}\left[(9 / 2)^{4}, v=4, \mathrm{~s}, J=4\right]
$$




$$
\begin{aligned}
= & -\frac{1}{3} E_{\mathrm{x}}\left[(9 / 2)^{2}, v=2, J=2\right]-\frac{1}{13} E_{\mathrm{x}}\left[(9 / 2)^{2}, v=2, J=4\right] \\
& +\frac{2}{15} E_{\mathrm{x}}\left[(9 / 2)^{2}, v=2, J=6\right]+\frac{18}{65} E_{\mathrm{x}}\left[(9 / 2)^{2}, v=2, J=8\right],
\end{aligned}
$$

associating the excitation energies of the $J=2,4,6$ and 8 , seniority $v=2$ states in the two-particle system with those of the $J=4$ and 6 , seniority $v=4$ states in the four-particle system.

Another interaction-independent result that can be derived concerns transition matrix elements between the two states. For example, an electric quadrupole transition between two states characterized by the expansion coefficients $\eta_{I_{\mathrm{i}} I_{\mathrm{i}}^{\prime}}$ and $\eta_{I_{\mathrm{f}} I_{\mathrm{f}}^{\prime}}$, as in Eq. (45), has the $B(\mathrm{E} 2)$ value

$$
\begin{aligned}
& B\left(\mathrm{E} 2 ; j^{4}\left\{\eta_{I_{\mathrm{i}} I_{\mathrm{i}}^{\prime}}\right\} J_{\mathrm{i}} \rightarrow j^{4}\left\{\eta_{I_{\mathrm{f}} I_{\mathrm{f}}^{\prime}}\right\} J_{\mathrm{f}}\right) \\
& =20\left(2 J_{\mathrm{f}}+1\right)(2 j+1) B\left(\mathrm{E} 2 ; 2_{1}^{+} \rightarrow 0_{1}^{+}\right) \\
& \times\left[\sum_{I_{\mathrm{i}} I_{\mathrm{i}}^{\prime}} \sum_{I_{\mathrm{f}} I_{\mathrm{f}}^{\prime}} \sum_{R_{\mathrm{i}} R_{\mathrm{f}} R^{\prime}} \eta_{I_{\mathrm{i}} I_{\mathrm{i}}^{\prime}} \sqrt{2 R_{\mathrm{i}}+1}\left[j^{2}\left(R_{\mathrm{i}}\right) j^{2}\left(R^{\prime}\right) ; J_{\mathrm{i}} \mid\right\} j^{4}\left[I_{\mathrm{i}} I_{\mathrm{i}}^{\prime}\right] J_{\mathrm{i}}\right]\left\{\begin{array}{ccc}
J_{\mathrm{i}} & R_{\mathrm{i}} & R^{\prime} \\
R_{\mathrm{f}} & J_{\mathrm{f}} & 2
\end{array}\right\} \\
& \left.\left.\times \eta_{I_{\mathrm{f}} I_{\mathrm{f}}^{\prime}} \sqrt{2 R_{\mathrm{f}}+1}\left[j^{2}\left(R_{\mathrm{f}}\right) j^{2}\left(R^{\prime}\right) ; J_{\mathrm{f}} \mid\right\} j^{4}\left[I_{\mathrm{f}} I_{\mathrm{f}}^{\prime}\right] J_{\mathrm{f}}\right]\left\{\begin{array}{ccc}
R_{\mathrm{i}} & j & j \\
j & R_{\mathrm{f}} & 2
\end{array}\right\}\right]^{2},
\end{aligned}
$$

where $B\left(\mathrm{E} 2 ; 2_{1}^{+} \rightarrow 0_{1}^{+}\right)$is the $B(\mathrm{E} 2)$ value in the two-particle system $j^{2}$. For the two solvable states in the $j=9 / 2$ four-particle system this reduces to the relation

$$
\begin{array}{r}
B\left(\mathrm{E} 2 ;(9 / 2)^{4}, v=4, \mathrm{~s}, J=6 \rightarrow(9 / 2)^{4}, v=4, \mathrm{~s}, J=4\right) \\
=\frac{209475}{176468} B\left(\mathrm{E} 2 ; 2_{1}^{+} \rightarrow 0_{1}^{+}\right) \approx 1.19 B\left(\mathrm{E} 2 ; 2_{1}^{+} \rightarrow 0_{1}^{+}\right),
\end{array}
$$

which defines an interaction-independent relation between the properties of the two- and four-particle systems.

We have searched for other examples of partial seniority conservation but failed to find any for half-odd-integer values $j \neq 9 / 2$. So it transpires that the two solvable seniority $v=4$ states of the four-particle system in the $j=9 / 2$ shell are unique. The situation for bosons is different, as discussed in Sect. 5 .

Although the mathematical derivation of the necessary conditions for the existence of partial seniority conservation is clear, a simple, intuitive reason for it is still lacking. In Ref. [25] some progress towards this goal has been made, and in particular a partial understanding with analytic arguments of the coefficients entering the energy expressions (51) has been achieved. So far, the best 
explanation of the anomalous partial conservation of seniority in the $j=9 / 2$ shell has been given by Qi [26] who found an analytic derivation of this property based on the uniqueness of the $\left|j^{5}, v=5, J=j\right\rangle$ state for $j=9 / 2$, state which is no longer unique for $j>9 / 2$.

\section{$5 \quad$ Partial seniority conservation for $N$ identical bosons}

In Sect. 2 are given the sufficient conditions (8) for a hamiltonian describing a system of interacting bosons or fermions to have a dynamical symmetry. The derivation is based on a simple counting argument which for bosons is as follows. For identical bosons the number of independent quadratic Casimir operators in the canonical classification (1) is two for $\ell=1$ and three for $\ell>1$. (In this section the notation $\ell$ instead of $j$ is used for the spin of the particles, to emphasize that they are bosons.) This matches the number of two-body interactions for $p$ and $d$ bosons which therefore are solvable systems. If the spin of the bosons exceeds $\ell=2$ ( $f$ bosons and beyond), there are more twobody interactions than quadratic Casimir operators, and a general two-body hamiltonian does not have a dynamical symmetry. In this section it is shown that in systems of interacting bosons with spin $\ell \geq 3$ many states occur to which additional constraints apply as a consequence of which they are solvable.

For the present discussion it is convenient to replace the quadratic Casimir operators in the hamiltonian (4) with equivalent operators that are of pure two-body character. The quadratic Casimir operator of $\mathrm{U}(n)$ (with $n \equiv 2 \ell+1$ ) is, up to a term linear in the boson number operator $\hat{N}$, equivalent to a constant interaction between the bosons which shall be denoted as $\hat{C}$. The quadratic Casimir operator of $\mathrm{SO}(n)$ is, up to linear and quadratic terms in $\hat{N}$, equivalent to a pairing interaction $\hat{P}$. Finally, the quadratic Casimir operator of $\mathrm{SO}(3)$ is identical to the square of the angular momentum operator $\hat{J}^{2}$; again it is more convenient to retain only its two-body part which shall be denoted as $\hat{J}_{\mathrm{tb}}^{2}$. In terms of the earlier defined two-body operators $\hat{V}_{\lambda}$, one has the identities

$$
\hat{C}=\sum_{\lambda} \hat{V}_{\lambda}, \quad \hat{P}=\hat{V}_{0}, \quad \hat{J}_{\mathrm{tb}}^{2}=\sum_{\lambda}[\lambda(\lambda+1)-2 \ell(\ell+1)] \hat{V}_{\lambda} .
$$

Given their connection with the quadratic Casimir operators in the classification (1), matrix elements of the operators $\hat{C}, \hat{P}$ and $\hat{J}_{\mathrm{tb}}^{2}$ can be found in closed form,

$$
\left\langle\ell^{N} v \alpha J|\hat{C}| \ell^{N} v \alpha J\right\rangle=\frac{1}{2} N(N-1),
$$




$$
\begin{aligned}
\left\langle\ell^{N} v \alpha J|\hat{P}| \ell^{N} v \alpha J\right\rangle & =N(N+n-2)-v(v+n-2), \\
\left\langle\ell^{N} v \alpha J\left|\hat{J}_{\mathrm{tb}}^{2}\right| \ell^{N} v \alpha J\right\rangle & =J(J+1)-N \ell(\ell+1) .
\end{aligned}
$$

Let us consider now a state $\left|\ell^{N} v \alpha J\right\rangle$ and ask the question whether values of boson number $N$, seniority $v$, multiplicity label $\alpha$ and angular momentum $J$ exist for which this is an eigenstate of an arbitrary two-body hamiltonian $\hat{V}=\sum_{\lambda} \nu_{\lambda} \hat{V}_{\lambda}$ with analytic eigenvalues of the form

$$
E\left(\ell^{N} v \alpha J\right)=\sum_{\lambda} a_{\lambda} \nu_{\lambda}
$$

in terms of the two-body matrix elements $\nu_{\lambda}$ with coefficients $a_{\lambda}$ that are functions of $\ell, N, v, \alpha$ and $J$. The analysis concerns states of maximum seniority, $v=N$. It has been remarked earlier that partial conservation of seniority occurs 'trivially' in a number of cases. The most obvious example is an $N$-boson state of stretched angular momentum $J=\ell N$. It is clear that this state must have seniority $v=\ell$ and that only the stretched interaction matrix element with $\lambda=2 \ell$ can contribute to its energy. Formally, this result is obtained from the expression for the matrix element in terms of $N$-to- $(N-2)$-particle CFPs,

$$
\begin{aligned}
\left\langle\ell^{N} v \alpha J\left|\hat{V}_{\lambda}\right| \ell^{N} v^{\prime} \alpha^{\prime} J\right\rangle=\frac{N(N-1)}{2} & \left.\sum_{v_{1} \alpha_{1} J_{1}}\left[\ell^{N-2}\left(v_{1} \alpha_{1} J_{1}\right) \ell^{2}(\lambda) ; J \mid\right\} \ell^{N} v \alpha J\right] \\
& \left.\times\left[\ell^{N-2}\left(v_{1} \alpha_{1} J_{1}\right) \ell^{2}(\lambda) ; J \mid\right\} \ell^{N} v^{\prime} \alpha^{\prime} J\right] .(
\end{aligned}
$$

For a state with $J=\ell N$ the intermediate state is unique with $v_{1}=N-2$ and $J_{1}=\ell(N-2)$, and the only interaction that couples $J_{1}$ to $J$ has $\lambda=2 \ell$. Since the corresponding CFP is unique it equals one and hence

$$
E\left(\ell^{N}, v=N, J=\ell N\right)=\frac{N(N-1)}{2} \nu_{2 \ell}
$$

This argument clearly is only appropriate for $J=\ell N$ and $v=N$. Similar but modified versions of it are possible for $J<\ell N$ and rely on the knowledge of the multiplicity $d_{v}^{(\ell)}(J)$, which specifies how many times the angular momentum $J$ occurs for a given seniority $v$. A closed formula is available for $d_{v}^{(\ell)}(J)$ in terms of an integral over characters of the orthogonal algebras $\mathrm{SO}(n)$ and $\mathrm{SO}(3)$, known from Weyl [27]. This leads to the following complex integral [28]:

$$
d_{v}^{(\ell)}(J)=\frac{i}{2 \pi} \oint_{|z|=1} \frac{\left(z^{2 J+1}-1\right)\left(z^{2 v+2 \ell-1}-1\right) \prod_{k=1}^{2 \ell-2}\left(z^{v+k}-1\right)}{z^{\ell v+J+2} \prod_{k=1}^{2 \ell-2}\left(z^{k+1}-1\right)} d z .
$$

By virtue of Cauchy's theorem the multiplicity $d_{v}^{(\ell)}(J)$ is obtained as the neg- 
ative of the residue of the integrand in Eq. (66).

To illustrate how multiplicity enters into the discussion of partial conservation of seniority, it is easier to specify a value for the boson spin $\ell$. Let us choose $\ell=3$. Multiplicities for $f$ bosons are given in Table 1 up to seniority $v=15$. No state exists with angular momentum $J=3 N-1$ and the highest possible, non-stretched angular momentum is $J=3 N-2$. This state is unique and must have seniority $v=N$ since $J=3 N-2$ does not occur for lower seniorities, that is, $d_{v}^{(f)}(3 N-2)=0$ for $v=N-2, N-4, \ldots$ The eigenvalue of this state can be found by noting from the expression (64) that the interaction $\hat{V}_{2}$ cannot contribute to its energy since the highest angular momentum of the intermediate $f^{N-2}$ system is $J_{1}=3(N-2)$ which cannot couple with $\lambda=2$ to $J=3 N-2$. Hence one establishes the equations

$$
\begin{aligned}
a_{0}=a_{2} & =0 \\
a_{0}+a_{2}+a_{4}+a_{6} & =\frac{1}{2} N(N-1), \\
-24 a_{0}-18 a_{2}-4 a_{4}+18 a_{6} & =(3 N-2)(3 N-1)-12 N,
\end{aligned}
$$

which can be solved to yield the energy expression

$$
E\left(f^{N}, v=N, J=3 N-2\right)=\frac{6 N-1}{11} \nu_{4}+\frac{11 N^{2}-23 N+2}{22} \nu_{6} .
$$

The next highest angular momentum $J=3 N-3$ is also unique and exists for $N \geq 3$. A closed energy expression can be found with the same argument,

$$
E\left(f^{N}, v=N, J=3 N-3\right)=\frac{9 N-3}{11} \nu_{4}+\frac{11 N^{2}-29 N+6}{22} \nu_{6} .
$$

For angular momentum $J=3 N-4$ one encounters the first case with multiplicity 2 (provided $N \geq 4$ ). The seniority of this state is still necessarily $v=N$ since $d_{v}^{(f)}(3 N-4)=0$ for $v=N-2, N-4, \ldots$ However, unlike the previous cases, it can couple with the interaction $\hat{V}_{2}$ to the stretched state of the intermediate $f^{N-2}$ system with angular momentum $J_{1}=3(N-2)$, so the interaction energy associated with $\hat{V}_{2}$ does not necessarily vanish but is given by

$$
\begin{array}{r}
\left\langle f^{N}, v=N, \alpha, J=3 N-4\left|\hat{V}_{2}\right| f^{N}, v=N, \alpha, J=3 N-4\right\rangle \\
\left.=\frac{N(N-1)}{2}\left[f^{N-2}\left(J_{1}=3 N-6\right) f^{2}(2) ; J \mid\right\} f^{N} v \alpha J\right]^{2} .
\end{array}
$$

There are two states with $J=3 N-4$ and $v=N$, characterized by $\alpha_{1}$ and $\alpha_{2}$, and only one intermediate state with $J_{1}=3 N-6$. One can therefore always 
Table 1

Multiplicity $d_{v}^{(f)}(J)$ for $f$ bosons up to seniority $v=15$.

\begin{tabular}{|c|c|c|c|c|c|c|c|c|c|c|c|c|c|c|c|}
\hline$J \backslash v$ & 1 & 2 & 3 & 4 & 5 & 6 & 7 & 8 & 9 & 10 & 11 & 12 & 13 & 14 & 15 \\
\hline 0 & 0 & 0 & 0 & 1 & 0 & 1 & 0 & 1 & 0 & 2 & 0 & 2 & 0 & 2 & 1 \\
\hline 1 & 0 & 0 & 1 & 0 & 1 & 0 & 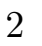 & & 2 & 1 & 3 & & 4 & 2 & 5 \\
\hline 2 & 0 & 1 & 0 & 1 & 1 & 2 & 1 & 3 & 2 & 4 & 3 & 5 & 4 & 7 & 5 \\
\hline 3 & 1 & 0 & 1 & 1 & 2 & 2 & 3 & 2 & 5 & 4 & 6 & 5 & 8 & 7 & 10 \\
\hline 4 & 0 & 1 & 1 & 2 & 1 & 3 & 3 & 5 & 4 & 6 & 6 & 9 & 8 & 11 & 10 \\
\hline 5 & 0 & 0 & 1 & 1 & 3 & 2 & 4 & 4 & 6 & 6 & 9 & 8 & 11 & 11 & 15 \\
\hline 6 & 0 & 1 & 1 & 2 & 2 & 4 & 4 & 6 & 6 & 9 & 8 & 12 & 12 & 15 & 15 \\
\hline 7 & 0 & 0 & 1 & 1 & 3 & 3 & 5 & 5 & 8 & 8 & 11 & 11 & 15 & 15 & 19 \\
\hline 8 & 0 & 0 & 0 & 2 & 2 & 4 & 4 & 7 & 7 & 10 & 11 & 14 & 14 & 19 & 19 \\
\hline 9 & 0 & 0 & 1 & 1 & 2 & 3 & 6 & 6 & 9 & 9 & 13 & 14 & 18 & 18 & 23 \\
\hline 10 & 0 & 0 & 0 & 1 & 2 & 4 & 4 & 7 & 8 & 12 & 12 & 16 & 17 & 22 & 23 \\
\hline 11 & 0 & 0 & 0 & 0 & 2 & 2 & 5 & 6 & 9 & 10 & 14 & 15 & 20 & 21 & 26 \\
\hline 12 & 0 & 0 & 0 & 1 & 1 & 3 & 4 & 7 & 8 & 12 & 13 & 18 & 19 & 24 & 26 \\
\hline 13 & 0 & 0 & 0 & 0 & 1 & 2 & 4 & 5 & 9 & 10 & 15 & 16 & 21 & 23 & 29 \\
\hline 14 & 0 & 0 & 0 & 0 & 0 & 2 & 3 & 6 & 7 & 11 & 13 & 18 & 20 & 26 & 27 \\
\hline 15 & 0 & 0 & 0 & 0 & 1 & 1 & 3 & 4 & 8 & 10 & 14 & 16 & 22 & 24 & 31 \\
\hline 16 & 0 & 0 & 0 & 0 & 0 & 1 & 2 & 5 & 6 & 10 & 12 & 18 & 20 & 26 & 29 \\
\hline 17 & 0 & 0 & 0 & 0 & 0 & 0 & 2 & 3 & 6 & 8 & 13 & 15 & 21 & 24 & 31 \\
\hline 18 & 0 & 0 & 0 & 0 & 0 & 1 & 1 & 3 & 5 & 9 & 11 & 16 & 19 & 26 & 29 \\
\hline 19 & 0 & 0 & 0 & 0 & 0 & 0 & 1 & 2 & 5 & 6 & 11 & 14 & 20 & 23 & 30 \\
\hline 20 & 0 & 0 & 0 & 0 & 0 & 0 & 0 & 2 & 3 & 7 & 9 & 14 & 17 & 24 & 28 \\
\hline 21 & 0 & 0 & 0 & 0 & 0 & 0 & 1 & 1 & 3 & 5 & 9 & 12 & 18 & 21 & 29 \\
\hline 22 & 0 & 0 & 0 & 0 & 0 & 0 & 0 & 1 & 2 & 5 & 7 & 12 & 15 & 22 & 26 \\
\hline 23 & 0 & 0 & 0 & 0 & 0 & 0 & 0 & 0 & 2 & 3 & 7 & 9 & 15 & 19 & 26 \\
\hline 24 & 0 & 0 & 0 & 0 & 0 & 0 & 0 & 1 & 1 & 3 & 5 & 10 & 13 & 19 & 23 \\
\hline 25 & 0 & 0 & 0 & 0 & 0 & 0 & 0 & 0 & 1 & 2 & 5 & 7 & 12 & 16 & 24 \\
\hline 26 & 0 & 0 & 0 & 0 & 0 & 0 & 0 & 0 & 0 & 2 & 3 & 7 & 10 & 16 & 20 \\
\hline 27 & 0 & 0 & 0 & 0 & 0 & 0 & 0 & 0 & 1 & 1 & 3 & 5 & 10 & 13 & 20 \\
\hline 28 & 0 & 0 & 0 & 0 & 0 & 0 & 0 & 0 & 0 & 1 & 2 & 5 & 7 & 13 & 17 \\
\hline 29 & 0 & 0 & 0 & 0 & 0 & 0 & 0 & 0 & 0 & 0 & 2 & 3 & 7 & 10 & 16 \\
\hline 30 & 0 & 0 & 0 & 0 & 0 & 0 & 0 & 0 & 0 & 1 & 1 & 3 & 5 & 10 & 14 \\
\hline 31 & 0 & 0 & 0 & 0 & 0 & 0 & 0 & 0 & 0 & 0 & 1 & 2 & 5 & 7 & 13 \\
\hline 32 & 0 & 0 & 0 & 0 & 0 & 0 & 0 & 0 & 0 & 0 & 0 & & 3 & 7 & 10 \\
\hline 33 & 0 & 0 & 0 & 0 & 0 & 0 & 0 & 0 & 0 & 0 & 1 & 1 & 3 & 5 & 10 \\
\hline 34 & 0 & 0 & 0 & 0 & 0 & 0 & 0 & 0 & 0 & 0 & 0 & 1 & 2 & 5 & 7 \\
\hline 35 & 0 & 0 & 0 & 0 & 0 & 0 & 0 & 0 & 0 & 0 & 0 & 0 & 2 & 3 & 7 \\
\hline 36 & 0 & 0 & 0 & 0 & 0 & 0 & 0 & 0 & 0 & 0 & 0 & & 1 & 3 & \\
\hline 37 & 0 & 0 & 0 & 0 & 0 & 0 & 0 & 0 & 0 & 0 & 0 & 0 & 1 & 2 & 5 \\
\hline 38 & 0 & 0 & 0 & 0 & 0 & 0 & 0 & 0 & 0 & 0 & 0 & 0 & 0 & 2 & 3 \\
\hline 39 & 0 & 0 & 0 & 0 & 0 & 0 & 0 & 0 & 0 & 0 & 0 & 0 & 1 & 1 & 3 \\
\hline 40 & 0 & 0 & 0 & 0 & 0 & 0 & 0 & 0 & 0 & 0 & 0 & & 0 & 1 & 2 \\
\hline 41 & 0 & 0 & 0 & 0 & 0 & 0 & 0 & 0 & 0 & 0 & 0 & 0 & 0 & 0 & 2 \\
\hline 42 & 0 & 0 & 0 & 0 & 0 & 0 & 0 & 0 & 0 & 0 & 0 & 0 & 0 & 1 & 1 \\
\hline 43 & 0 & 0 & 0 & 0 & 0 & 0 & 0 & 0 & 0 & 0 & 0 & 0 & 0 & 0 & 1 \\
\hline 44 & 0 & 0 & 0 & 0 & 0 & 0 & 0 & 0 & 0 & 0 & 0 & 0 & 0 & 0 & 0 \\
\hline 45 & 0 & 0 & 0 & 0 & 0 & 0 & 0 & 0 & 0 & 0 & 0 & 0 & 0 & 0 & 1 \\
\hline
\end{tabular}

choose a linear combination of $\alpha_{1}$ and $\alpha_{2}$, say $\bar{\alpha}$, such that

$$
\left.\left[f^{N-2}\left(J_{1}=3 N-6\right) f^{2}(2) ; J \mid\right\} f^{N} v \bar{\alpha} J\right]=0 .
$$


This state satisfies

$$
\left\langle f^{N}, v=N, \alpha_{i}, J=3 N-4\left|\hat{V}_{2}\right| f^{N}, v=N, \bar{\alpha}, J=3 N-4\right\rangle=0,
$$

and by the same argument as before one can derive its energy in closed form,

$$
E\left(f^{N}, v=N, \bar{\alpha}, J=3 N-4\right)=\frac{12 N-6}{11} \nu_{4}+\frac{11 N^{2}-35 N+12}{22} \nu_{6} .
$$

The next highest angular momentum $J=3 N-5$ has also multiplicity 2 for $N \geq 5$. A closed energy expression can be found with the same argument,

$$
E\left(f^{N}, v=N, \bar{\alpha}, J=3 N-5\right)=\frac{15 N-10}{11} \nu_{4}+\frac{11 N^{2}-41 N+20}{22} \nu_{6} .
$$

The next case with angular momentum $J=3 N-6$ presents the additional complication that its seniority quantum number is not unique but can be $v=N$ or $v=N-2$. It can still be dealt with in the following way. There are two intermediate states in the expression for the matrix element of $\hat{V}_{2}$,

$$
\begin{gathered}
\left\langle f^{N}, v=N, \alpha, J=3 N-6\left|\hat{V}_{2}\right| f^{N}, v=N, \alpha, J=3 N-6\right\rangle \\
\left.=\frac{N(N-1)}{2} \sum_{J_{1}}\left[f^{N-2}\left(J_{1}\right) f^{2}(2) ; J \mid\right\} f^{N} v \alpha J\right]^{2},
\end{gathered}
$$

with $J_{1}=3 N-6$ or $3 N-8$. However, the state with angular momentum $J=3 N-6$ has multiplicity 3 (for $N \geq 6$ ) and hence a linear combination $\bar{\alpha}$ can always be chosen such that

$$
\left.\left[f^{N-2}\left(J_{1}\right) f^{2}(2) ; J \mid\right\} f^{N} v \bar{\alpha} J\right]=0, \quad \text { for } \quad J_{1}=3 N-6,3 N-8 .
$$

As a consequence, the contribution of $\hat{V}_{2}$ to the energy of the state $\mid f^{N}, v=$ $N, \bar{\alpha}, J=3 N-6\rangle$ vanishes. In addition, one has from Eq. (64) and the vanishing CFPs (76) that

$$
\left\langle f^{N}, v=N-2, J=3 N-6\left|\hat{V}_{2}\right| f^{N}, v=N, \bar{\alpha}, J=3 N-6\right\rangle=0,
$$

that is, the state does not mix with the state with seniority $v=N-2$. This is thus a first example of 'non-trivial' conservation of seniority since one has for a given angular momentum $J$ several possible seniorities $v$ and one state with seniority $v=N$ that does not mix with states of lower seniority. Its eigenvalue is found to be

$$
E\left(f^{N}, v=N, \bar{\alpha}, J=3 N-6\right)=\frac{18 N-15}{11} \nu_{4}+\frac{11 N^{2}-47 N+30}{22} \nu_{6} .
$$


The arguments for finding states which conserve seniority while all others do not, become increasingly complex but are still valid for the next two cases with angular momentum $J=3 N-7(N \geq 7)$ and $J=3 N-8(N \geq 8)$. The eigenvalue expressions are

$$
E\left(f^{N}, v=N, \bar{\alpha}, J=3 N-7\right)=\frac{21 N-21}{11} \nu_{4}+\frac{11 N^{2}-53 N+42}{22} \nu_{6},
$$

and

$$
E\left(f^{N}, v=N, \bar{\alpha}, J=3 N-8\right)=\frac{24 N-28}{11} \nu_{4}+\frac{11 N^{2}-59 N+56}{22} \nu_{6} .
$$

The preceding analysis can be generalized to bosons with any spin $\ell$. The solvable states satisfy the following energy expression:

$$
E\left(\ell^{N}, v=N, \bar{\alpha}, J=\ell N-q\right)=a_{N q}^{\ell} \nu_{2 \ell-2}+b_{N q}^{\ell} \nu_{2 \ell}
$$

with

$$
\begin{aligned}
a_{N q}^{\ell} & =\frac{q \ell N-(q-1) q}{4 \ell-1}, \\
b_{N q}^{\ell} & =\frac{(4 \ell-1) N^{2}-[(4 \ell-1)+2 q \ell] N+(q-1) q}{2(4 \ell-1)} .
\end{aligned}
$$

According to this analysis, partial conservation of seniority is restricted to states with maximal seniority, $v=N$, and occurs at the high end of allowed angular momenta. The surprising aspect of the result (82) is that partial conservation of seniority persists for high values of $q$, up to $q=8$.

\section{Applications in fermionic systems}

Many studies exist which show the relevance of seniority in nuclei. It is not the intention here to review all such applications, many of which are discussed in Ref. [5]. Rather, two consequences of the partial conservation of seniority are pointed out, which are related to the existence of seniority isomers and to properties of one-nucleon transfer. 


\subsection{Seniority isomers}

Isomers are metastable quantum states. In nuclei isomers generally adopt a configuration which is different from those of states at lower energy and their decay is therefore hindered. The type of configuration change determines the nature of the isomer and hence one distinguishes, for example, shape isomers, spin isomers and $K$ isomers [29].

Seniority isomers exist by virtue of seniority and its associated selection rules. Generally, and in particular in semi-magic nuclei, states with low seniority occur at low energy. For example, the ground state of an even-even semimagic nucleus has approximately seniority $v \approx 0$ (all nucleons in pairs coupled to $J=0$ ) while its yrast levels with angular momenta $J=2,4,6, \ldots$ have seniority $v \approx 2$ (containing one 'broken' pair with $J \neq 0$ ). Seniority isomerism is expected to occur in semi-magic nuclei because electric quadrupole (E2) transitions between states with seniority $v=2$ are small when the valence shell is close to half-filled. This result is a consequence of the fact that the matrix elements of even tensor operators - and hence also of the quadrupole operator-between states with seniority $v=2$ vanish at mid-shell $[4,5]$.

Examples of seniority isomers have been found in the $N=50$ isotones with protons dominantly confined to the $\pi 1 g_{9 / 2}$ shell [30]. In particular, the $J^{\pi}=8^{+}$ levels in ${ }^{92} \mathrm{Mo}(Z=42),{ }^{94} \mathrm{Ru}(Z=44),{ }^{96} \mathrm{Pd}(Z=46)$ and ${ }^{98} \mathrm{Cd}(Z=$ 48) have half-lives of $0.190(3), 71(4), 2.10(21)$ and $0.48(16) \mu \mathrm{s}$, respectively, resulting from a combination of slow E2 decay and a small energy difference with the $J^{\pi}=6^{+}$level below it. A review is given by Grawe et al. [31]. On the basis of similar arguments one would expect the same phenomenon to occur in the neutron-rich nickel $(Z=28)$ isotopes from ${ }^{70} \mathrm{Ni}$ to ${ }^{76} \mathrm{Ni}$ with neutrons dominantly confined to the $\nu 1 g_{9 / 2}$ shell. Isomers with $J^{\pi}=8^{+}$are indeed observed in ${ }^{70} \mathrm{Ni}$ and ${ }^{76} \mathrm{Ni}$ with half-lives of $0.232(1)$ and $0.59_{-11}^{+18} \mu \mathrm{s}$, respectively, but, in spite of intensive searches [32], none was found so far in ${ }^{72} \mathrm{Ni}$ or ${ }^{74} \mathrm{Ni}$. Only recently, a possible $J^{\pi}=\left(8^{+}\right)$level was identified in ${ }^{72} \mathrm{Ni}$ at an excitation energy $2590 \mathrm{keV}$, decaying to a $J^{\pi}=\left(6^{+}\right)$level with the emission of a 199-keV gamma [33]; only an upper limit of 20 ns could be determined for the half-life which therefore seems to exclude the isomeric character of this level.

An explanation of these observations is given in this subsection. The problem was discussed by Grawe et al. [34] who noted that the disappearance of the isomers in ${ }^{72} \mathrm{Ni}$ and ${ }^{74} \mathrm{Ni}$ is related to an inversion of levels with seniority $v=2$ and $v=4$. It will be shown that an analytic explanation exists on the basis of the results derived in Sect. 4 .

Let us begin with a discussion of the nickel isotopes from ${ }^{70} \mathrm{Ni}$ to ${ }^{76} \mathrm{Ni}$; since 


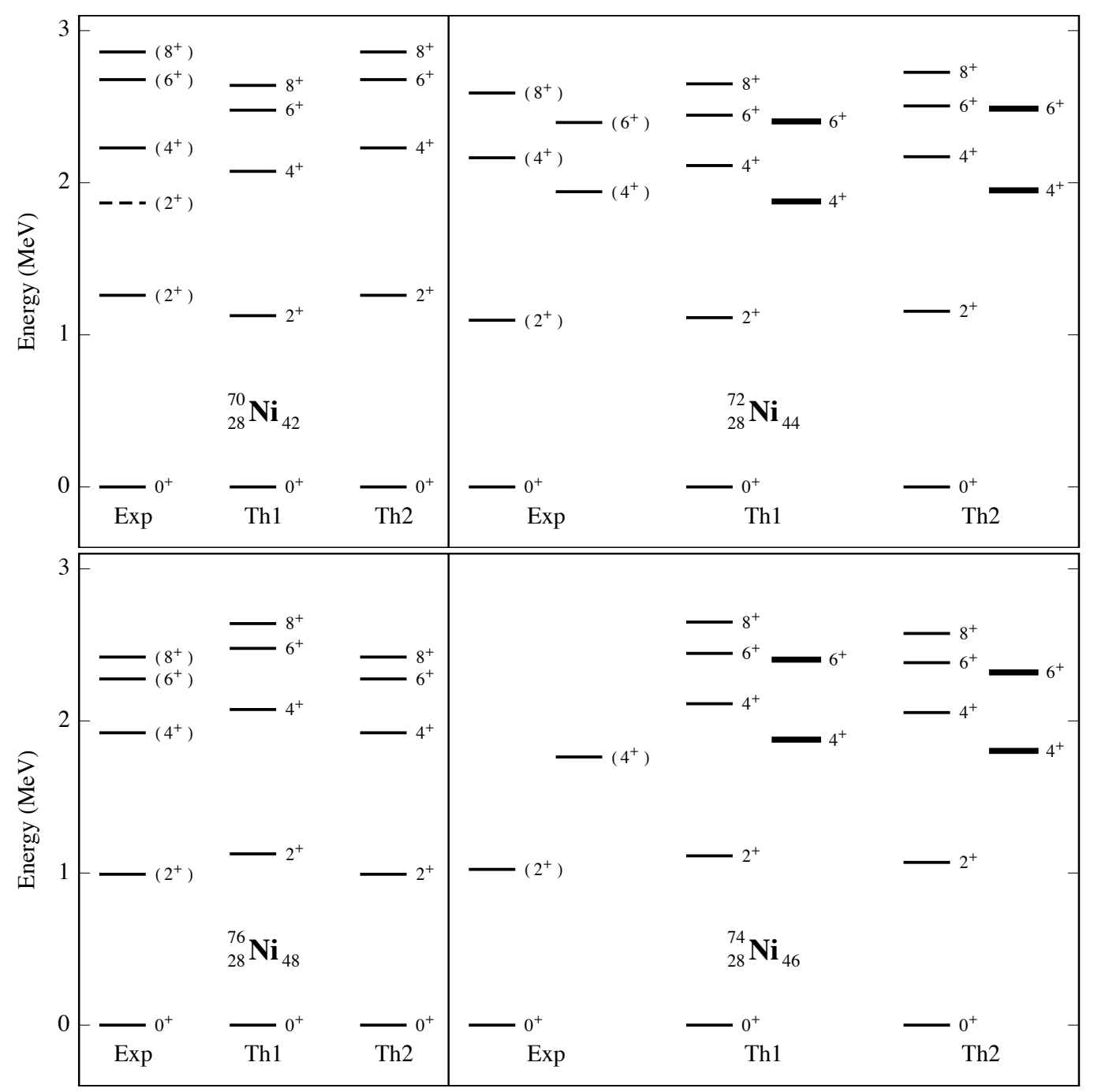

Fig. 2. The low-energy spectra of the nickel isotopes ${ }^{70-76} \mathrm{Ni}$. The left-hand columns 'Exp' show the observed levels while the columns 'Th1' and 'Th2' contain the results of a $\left(\nu 1 g_{9 / 2}\right)^{n}$ shell-model calculation with constant or linearly varying two-body matrix elements, respectively. The two solvable $J^{\pi}=4^{+}$and $J^{\pi}=6^{+}$states with seniority $v=4$ are shown with thick lines; the dashed line corresponds to an intruder level.

virtually nothing is known about the odd-mass nuclei, let us concentrate on the even-even ones. The left-hand columns of the spectra in Fig. 2 show the observed levels $[33,35,36]$. The nucleus ${ }^{70} \mathrm{Ni}$ displays, in a single-shell approximation, a two-neutron-particle spectrum $\left(\nu 1 g_{9 / 2}\right)^{2}$ with excited states with $J^{\pi}=2^{+}, 4^{+}, 6^{+}$and $8^{+}$. This is indeed found to be the case except for the additional $J^{\pi}=\left(2^{+}\right)$level at $1867 \mathrm{keV}$. This level is equally absent in shellmodel calculations in a large basis consisting of the $2 p_{1 / 2}, 2 p_{3 / 2}, 1 f_{5 / 2}$ and $1 g_{9 / 2}$ shells for neutrons and protons [37]. A possible explanation of the $1867 \mathrm{keV}$ level would therefore seem to require the $1 f_{7 / 2}$ shell and, in particular, proton excitations across the $Z=28$ shell gap might lead to low-lying states in ${ }^{70} \mathrm{Ni}$. The nucleus ${ }^{76} \mathrm{Ni}$ displays a two-neutron-hole spectrum $\left(\nu 1 g_{9 / 2}\right)^{-2}$ with the 
same yrast sequence as in ${ }^{70} \mathrm{Ni}$. The two-particle and the two-hole spectra fix the two-body matrix elements $\nu_{\lambda}$, or rather the differences $\nu_{\lambda}-\nu_{0}$. This is done at two levels of sophistication by taking either constant matrix elements that are the average of those in ${ }^{70} \mathrm{Ni}$ and ${ }^{76} \mathrm{Ni}$ (Th1) or by letting them vary linearly from ${ }^{70} \mathrm{Ni}$ to ${ }^{76} \mathrm{Ni}$ (Th2). In the latter approximation the spectra of the two-particle and the two-hole nuclei are exactly reproduced [except for the intruder $J^{\pi}=\left(2^{+}\right)$state in ${ }^{70} \mathrm{Ni}$; the description of the two intermediate isotopes, ${ }^{72} \mathrm{Ni}$ and ${ }^{74} \mathrm{Ni}$, should be rather accurate, albeit very empirical.

The two solvable $J^{\pi}=4^{+}$and $J^{\pi}=6^{+}$levels with seniority $v=4$ are shown with thick lines in Fig. 2. A noteworthy feature of the calculated spectra of ${ }^{72,74} \mathrm{Ni}$ is the occurrence of two levels for $J^{\pi}=4^{+}$and for $J^{\pi}=6^{+}$which are very close in energy, especially in the latter case. This is a direct consequence of the solvability of one member of each doublet which cannot mix with the close-lying, predominantly $v=2$ state with the same spin. At least for the $J^{\pi}=6^{+}$levels, this feature is still clearly present in the large-scale shell-model calculations of Lisetskiy et al. [37].

A similar analysis in the same approximation can be performed for the $N=50$ isotones with protons in the $\pi 1 g_{9 / 2}$ shell. In Fig. 3 the results of the calculation are compared with the observed spectra $[38,39,40,41]$. In ${ }^{94} \mathrm{Ru}\left(4_{2}^{+}\right)$and $\left(6_{2}^{+}\right)$ levels are observed at energies of 2503 and $2818 \mathrm{keV}$, respectively [39]; these are possible candidates for the solvable states which are calculated at 2422 and $2828 \mathrm{keV}$ in the approximation 'Th2'. It would be of interest to confirm the spin assignment and to attempt to measure the E2 transition probability between the two levels.

There is a striking difference between the calculated four-particle (or fourhole) spectra of the nickel isotopes and those of the $N=50$ isotones: the solvable $J^{\pi}=4^{+}$and $J^{\pi}=66^{+}$states are yrast in ${ }^{72} \mathrm{Ni}$ and ${ }^{74} \mathrm{Ni}$ while they are yrare in ${ }^{94} \mathrm{Ru}$ and ${ }^{96} \mathrm{Pd}$. For this reason one may conjecture that the observed yrast $\left(4^{+}\right)$and $\left(6^{+}\right)$levels in ${ }^{72} \mathrm{Ni}$ and the observed yrast $\left(4^{+}\right)$level in ${ }^{74} \mathrm{Ni}$ are the solvable states in question, as is done in Fig. 2.

For any reasonable interaction between identical nucleons in a $j=9 / 2$ shell, the seniority classification is a good approximation. This was shown to be true for specific cases by Grawe et al. [34] but can be argued from general considerations. Consider as an example the $J^{\pi}=0^{+}$states. There are two $J^{\pi}=0^{+}$states for four particles in a $j=9 / 2$ shell with seniority $v=0$ and $v=4$, respectively. Introducing the notation $\left|0_{v=0}^{+}\right\rangle \equiv\left|(9 / 2)^{4}, v=0, J=0\right\rangle$ and $\left|0_{v=4}^{+}\right\rangle \equiv\left|(9 / 2)^{4}, v=4, J=0\right\rangle$, one finds with the help of the results of Sect. 4 the following matrix elements:

$$
\left\langle 0_{v=0}^{+}|\hat{V}| 0_{v=0}^{+}\right\rangle=\frac{8}{5} \nu_{0}+\frac{1}{2} \nu_{2}+\frac{9}{10} \nu_{4}+\frac{13}{10} \nu_{6}+\frac{17}{10} \nu_{8}
$$




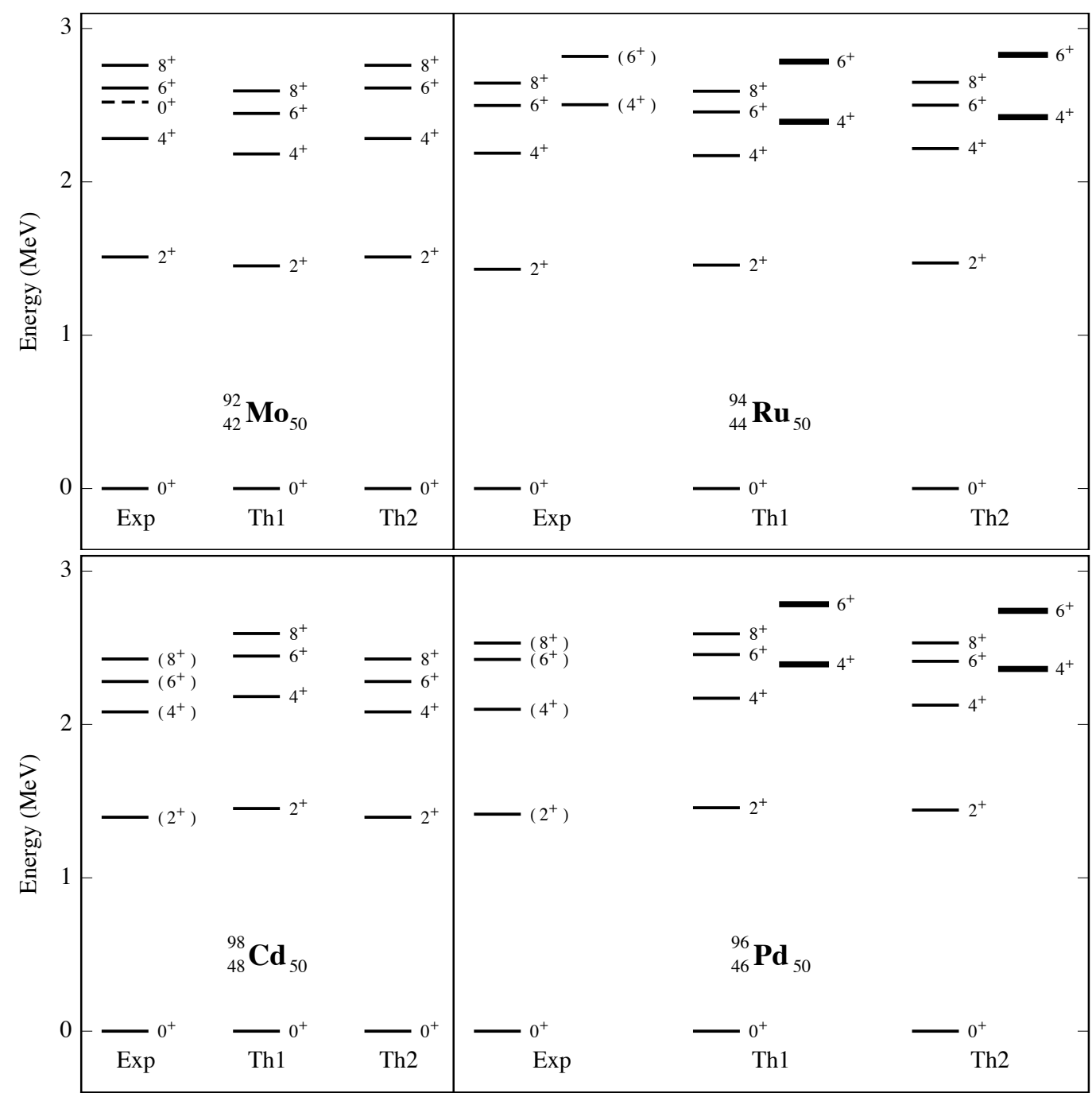

Fig. 3. The low-energy spectra of the $N=50$ isotones ${ }^{92} \mathrm{Mo},{ }^{94} \mathrm{Ru},{ }^{96} \mathrm{Pd}$ and ${ }^{98} \mathrm{Cd}$. The left-hand columns 'Exp' show the observed levels while the columns 'Th1' and 'Th2' contain the results of a $\left(\pi 1 g_{9 / 2}\right)^{n}$ shell-model calculation with constant or linearly varying two-body matrix elements, respectively. The two solvable $J^{\pi}=4^{+}$ and $J^{\pi}=6^{+}$states with seniority $v=4$ are shown with thick lines; the dashed line corresponds to an intruder level.

$$
\begin{aligned}
\left\langle 0_{v=0}^{+}|\hat{V}| 0_{v=4}^{+}\right\rangle & =\frac{-65 \nu_{2}+315 \nu_{4}-403 \nu_{6}+153 \nu_{8}}{10 \sqrt{429}} \\
\left\langle 0_{v=4}^{+}|\hat{V}| 0_{v=4}^{+}\right\rangle & =\frac{13}{66} \nu_{2}+\frac{735}{286} \nu_{4}+\frac{961}{330} \nu_{6}+\frac{459}{1430} \nu_{8} .
\end{aligned}
$$

The extent of the breaking of seniority depends on the size of the off-diagonal matrix element divided by the difference between the diagonal ones. For any reasonable choice of interaction this ratio is small. For example, for any of the interactions fitted to the nickel isotopes or the $N=50$ isotones, the differences between the exact and the diagonal energies are less than $2 \mathrm{keV}$ and the admixtures of seniority $v=4$ in the ground state do not exceed $0.1 \%$ 
in amplitude. A similar argument applies to the $J^{\pi}=2^{+}$and $J^{\pi}=8^{+}$states.

The proof that seniority mixing is negligible for all $J^{\pi}=4^{+}$and $J^{\pi}=6^{+}$states of a four-particle $j=9 / 2$ system is more subtle. There are three states for each of these angular momenta, two of which, with seniority $v=2$ and $v=4$, are close in energy and could possibly strongly mix. However, the seniority $v=4$ members of the closely-spaced doublets are precisely the solvable $J^{\pi}=4^{+}$ and $J^{\pi}=6^{+}$states discussed in Sect. 4, and they conserve seniority for any interaction. As a consequence, breaking of seniority only arises through mixing of the seniority $v=2$ and the higher-lying seniority $v=4$ state and, by the same argument as above, this mixing is found to be small.

The conclusion of this discussion is that, for any reasonable two-body interaction, seniority is a good quantum number for all states in a $j=9 / 2$ shell. Exact energies obtained from a diagonalization are close to the approximate seniority formulas, which for the $J^{\pi}=4^{+}$and $J^{\pi}=6^{+}$states with seniority $v=2$ are given by

$$
\begin{aligned}
& E\left[(9 / 2)^{4}, v=2, J=4\right]=\frac{3}{5} \nu_{0}+\frac{67}{99} \nu_{2}+\frac{746}{715} \nu_{4}+\frac{1186}{495} \nu_{6}+\frac{918}{715} \nu_{8}, \\
& E\left[(9 / 2)^{4}, v=2, J=6\right]=\frac{3}{5} \nu_{0}+\frac{34}{99} \nu_{2}+\frac{1186}{715} \nu_{4}+\frac{658}{495} \nu_{6}+\frac{1479}{715} \nu_{8} .
\end{aligned}
$$

Comparison of these expressions with the corresponding ones for the solvable $J^{\pi}=4^{+}$and $J^{\pi}=6^{+}$states with seniority $v=4$, Eq. (51), makes it clear that the lowering of the solvable states in ${ }^{72,74} \mathrm{Ni}$ is associated with the low excitation energy of the $J^{\pi}=2^{+}$level in the two-particle and two-hole nuclei ${ }^{70} \mathrm{Ni}$ and ${ }^{76} \mathrm{Ni}$. In the corresponding $N=50$ isotones, ${ }^{92} \mathrm{Mo}$ and ${ }^{98} \mathrm{Cd}$, the $J^{\pi}=2^{+}$level is at higher energy and, because the coefficient of $\nu_{2}$ in Eq. (51) is larger than the one in Eq. (84), this results in a higher excitation energy of both solvable states in ${ }^{94} \mathrm{Ru}$ and ${ }^{96} \mathrm{Pd}$.

Partial seniority conservation sheds also some new light on the existence of seniority isomers [24,42]. Figure 4 illustrates the E2 decay for four particles in the $j=9 / 2$ shell as obtained with a seniority-conserving interaction. On the left-hand side it shows the 'typical' decay with very small $B(\mathrm{E} 2)$ values between states with seniority $v=2$ which is characteristic of the seniority classification in nuclei near mid-shell $(N \approx j+1 / 2)$ and which is at the basis of the explanation of seniority isomers [31]. This situation applies to ${ }^{94} \mathrm{Ru}$ and ${ }^{96} \mathrm{Pd}$ where the states with seniority $v=2$ are yrast. Another condition, necessary for the existence of the $J^{\pi}=8^{+}$state as an isomer, is that it should occur below the solvable $J^{\pi}=6^{+}$, which can be easily verified by comparing its energy, Eq. (51), with the one of the $J^{\pi}=8^{+}$state, in a seniority 


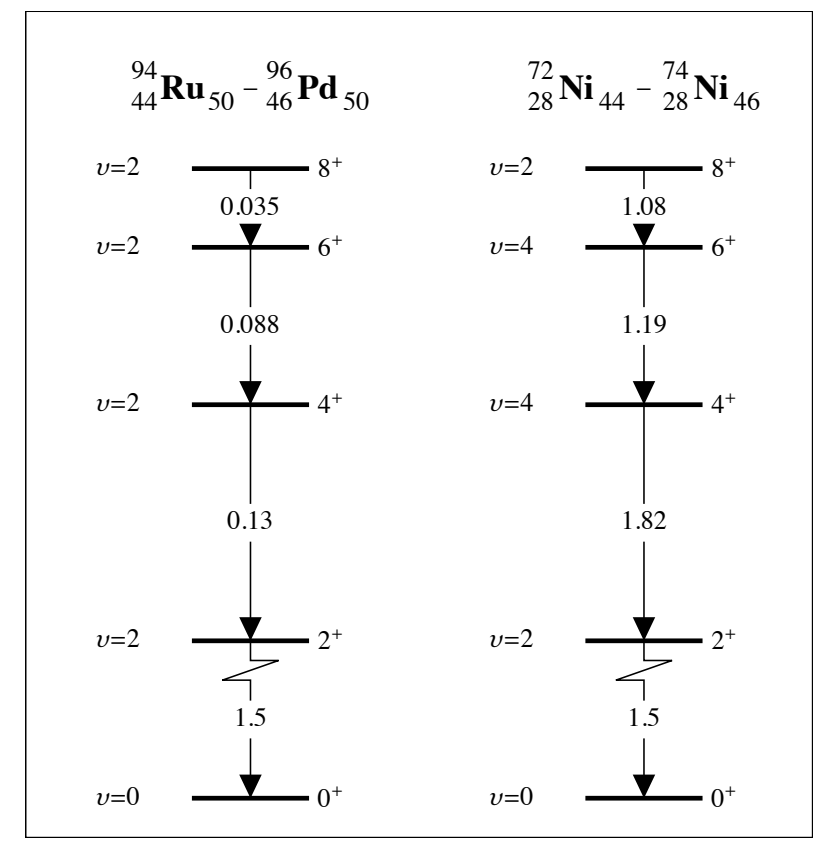

Fig. 4. E2 decay in the $(9 / 2)^{4}$ system as expected in the $N=50$ isotones (left) and the $Z=28$ nickel isotopes (right). The numbers between the levels denote $B(\mathrm{E} 2)$ values expressed in units of $B\left(\mathrm{E} 2 ; 2_{1}^{+} \rightarrow 0_{1}^{+}\right)$of the two-particle system and obtained with a seniority-conserving interaction.

approximation given by

$$
E\left[(9 / 2)^{4}, v=2, J=8\right]=\frac{3}{5} \nu_{0}+\frac{6}{11} \nu_{2}+\frac{486}{715} \nu_{4}+\frac{87}{55} \nu_{6}+\frac{1854}{715} \nu_{8}
$$

On the right-hand side of Fig. 4 is shown the E2 decay pattern as it is calculated in ${ }^{72,74} \mathrm{Ni}$. As argued above, it can be expected in these isotopes that the yrast $J^{\pi}=4^{+}$and $J^{\pi}=6^{+}$levels have seniority $v=4$, and this drastically alters the E2 decay pattern in the yrast band. As a consequence, unless the $J^{\pi}=8^{+}$and $J^{\pi}=6^{+}$levels are very close in energy, the former is unlikely to be isomeric.

The results of this analysis are schematically summarized in Fig. 5 which shows the evolution of the two- and four-particle spectra as a function of $R(4 / 2)$, the ratio of excitation energies of the yrast $J^{\pi}=4^{+}$and $J^{\pi}=2^{+}$ levels in the two-particle spectrum. This ratio is assumed in the figure to vary between two extreme values, $R(4 / 2)=1$ and $R(4 / 2)=2$, beyond which a seniority classification certainly is not any longer a reasonable approximation. The excitation energies of the yrast levels in the two-particle spectrum with $J^{\pi}=4^{+}, J^{\pi}=6^{+}$and $J^{\pi}=8^{+}$are assumed to be constant. In the upper panel of Fig. 5 is shown the evolution of levels of the four-particle spectrum as a function of $R(4 / 2)$ in the two-particle spectrum. If effects of seniority mixing (which are small) are neglected, excitation energies vary linearly with 


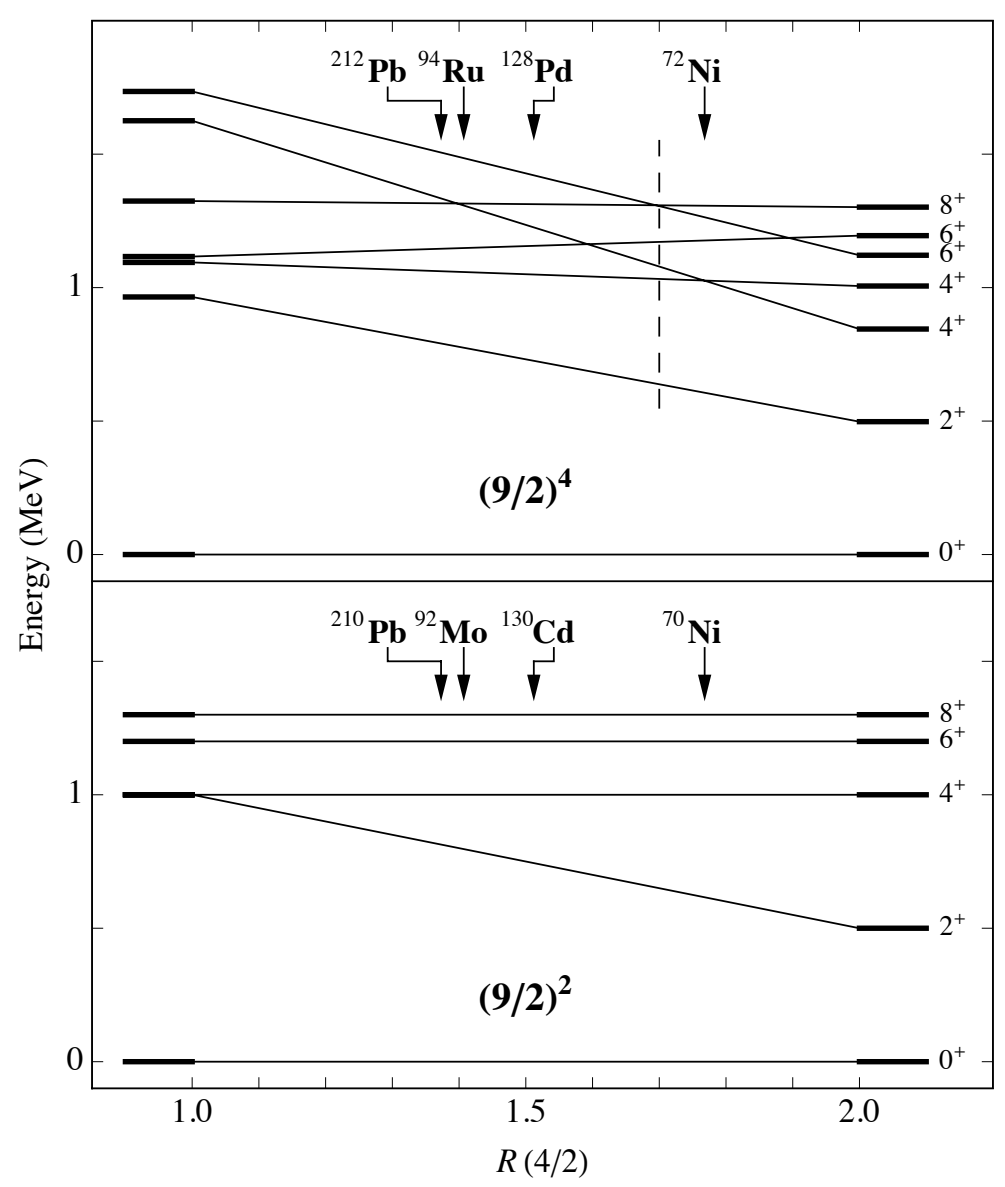

Fig. 5. Schematic illustration of the effect of $R(4 / 2)$, the ratio of excitation energies of the yrast $J^{\pi}=4^{+}$and $J^{\pi}=2^{+}$levels in the two-particle spectrum (bottom), on the properties of the four-particle spectrum (top). The arrows indicate the positions of a number of isotopes according to their $R(4 / 2)$ ratio in the two-particle (or two-hole) spectra, and the corresponding four-particle (or four-hole) isotopes.

$R(4 / 2)$. Not surprisingly, the excitation energy of the $J^{\pi}=2^{+}$level in the four-particle spectrum approximately drops by a factor two as $R(4 / 2)$ changes from 1 to 2 but the other levels with seniority $v=2$ remain approximately constant in energy. The biggest change, however, occurs for the two solvable levels with seniority $v=4$ to the extent that they cross some of the levels with seniority $v=2$. The dashed line indicates the point where the $J^{\pi}=6^{+}$ level with seniority $v=4$ crosses the $J^{\pi}=8^{+}$level with seniority $v=2$; the latter level is expected to be isomeric for smaller values of $R(4 / 2)$. The arrows indicate the positions on this diagram of a number of isotopes according to their $R(4 / 2)$ ratio in the two-particle (or two-hole) spectra. Extrapolation to the four-particle (or four-hole) spectra then leads to the conclusion that no $J^{\pi}=8^{+}$isomer should exist in ${ }^{72} \mathrm{Ni}$ (nor in ${ }^{74} \mathrm{Ni}$ ) while they should occur in ${ }^{212} \mathrm{~Pb}$ (and ${ }^{214} \mathrm{~Pb}$ ), ${ }^{94} \mathrm{Ru}$ (and ${ }^{96} \mathrm{Pd}$ ), as well as in ${ }^{128} \mathrm{Pd}$ (and ${ }^{126} \mathrm{Ru}$ ).

In a less schematic analysis the exact positions of the $J^{\pi}=6^{+}$and $J^{\pi}=8^{+}$ 


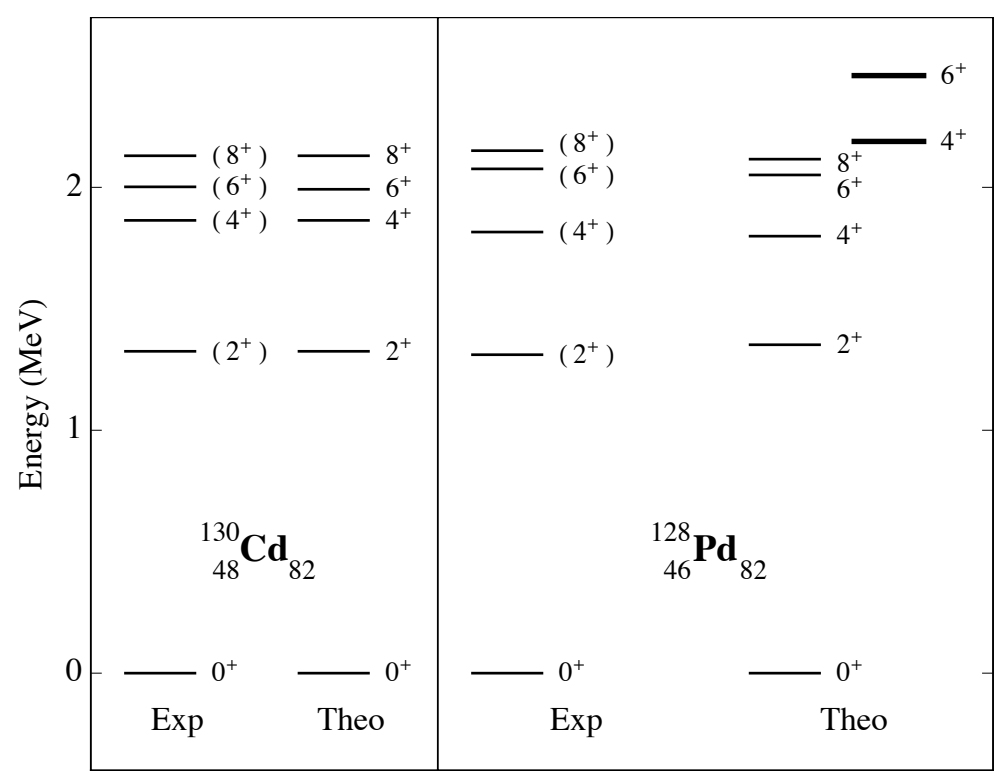

Fig. 6. The low-energy spectra of the isotopes ${ }^{130} \mathrm{Cd}$ and ${ }^{128} \mathrm{Pd}$. The left-hand columns 'Exp' show the observed levels while the columns 'Theo' contain the results of a $\left(\pi 1 g_{9 / 2}\right)^{n}$ shell-model calculation with constant two-body matrix elements. The two solvable $J^{\pi}=4^{+}$and $J^{\pi}=6^{+}$states with seniority $v=4$ are shown with thick lines.

levels of the two-particle spectrum should be taken instead of the plausible but somewhat arbitrary values of Fig. 5. The influence of these energies in this analysis is weak, however, and the same conclusion is obtained with the correct energies, that is, the $J^{\pi}=8^{+}$levels of the four-particle (or four-hole) nuclei are isomeric except those in ${ }^{72} \mathrm{Ni}$ and ${ }^{74} \mathrm{Ni}$.

Besides the nickel isotopes and the $N=50$ isotones, already discussed in the preceding paragraphs, Fig. 5 predicts properties of the neutron-rich lead isotopes $(Z=82)$ and the proton-poor $N=82$ isotones. The two-particle nucleus ${ }^{210} \mathrm{~Pb}$ is particularly stiff, characterized by a low ratio $R(4 / 2)=1.37$ and therefore $J^{\pi}=8^{+}$seniority isomers should exist in ${ }^{212} \mathrm{~Pb}$ and ${ }^{214} \mathrm{~Pb}$. This is indeed confirmed by recent experiments [43].

Another interesting application concerns the existence of a $J^{\pi}=8^{+}$seniority isomer in ${ }^{128} \mathrm{Pd}$. An isomer in this extremely neutron-rich palladium isotope $(Z=46)$ was detected at the RIBF facility at RIKEN with a delayedcoincidence technique between the identified radioactive ion and gamma rays de-exciting the isomeric state after passing through the spectrometer [44]. The two-hole spectrum of ${ }^{130} \mathrm{Cd}$ is well known and contains a $J^{\pi}=8^{+}$isomer with a half-life of $0.22(3) \mu \mathrm{s}$ [45]. On the basis of these properties of ${ }^{130} \mathrm{Cd}$, a simple prediction can be made of those of ${ }^{128} \mathrm{Pd}$ (see Fig. 6) with a $J^{\pi}=8^{+}$level at $2116 \mathrm{keV}$ which turns out to be isomeric since it occurs below the $J^{\pi}=6^{+}$ state with seniority $v=4$ at $2460 \mathrm{keV}$ and just above the $J^{\pi}=6^{+}$state with seniority $v=2$ at $2051 \mathrm{keV}$. The half-life $T_{1 / 2}\left({ }^{128} \mathrm{Pd} ; 8_{1}^{+}\right)$of the $J^{\pi}=8^{+}$level 
in ${ }^{128} \mathrm{Pd}$ can be estimated from

$$
\frac{T_{1 / 2}\left({ }^{128} \mathrm{Pd} ; 8_{1}^{+}\right)}{T_{1 / 2}\left({ }^{130} \mathrm{Cd} ; 8_{1}^{+}\right)}=\frac{1+\alpha_{\mathrm{Cd}}(128)}{1+\alpha_{\mathrm{Pd}}(75)}\left(\frac{128}{75}\right)^{5} \frac{B\left(\mathrm{E} 2 ; 8_{1}^{+} \rightarrow 6_{1}^{+}\right)^{130} \mathrm{Cd}}{B\left(\mathrm{E} 2 ; 8_{1}^{+} \rightarrow 6_{1}^{+}\right)^{128} \mathrm{Pd}}
$$

where $\alpha_{\mathrm{X}}\left(E_{\gamma}\right)$ is the internal electron conversion coefficient for a transition with energy $E_{\gamma}$ (in $\mathrm{keV}$ ) in the element $\mathrm{X}$. The gamma-ray energies for the $8_{1}^{+} \rightarrow 6_{1}^{+}$transitions in ${ }^{128} \mathrm{Pd}$ and ${ }^{130} \mathrm{Cd}, E_{\gamma}=75$ and 128 , are taken from experiment $[44,45]$. In the seniority scheme the following relations are valid (see Fig. 4):

$$
\begin{aligned}
& B\left(\mathrm{E} 2 ; 8_{1}^{+} \rightarrow 6_{1}^{+}\right){ }^{130} \mathrm{Cd}=0.318 B\left(\mathrm{E} 2 ; 2_{1}^{+} \rightarrow 0_{1}^{+}\right)^{130} \mathrm{Cd} \\
& B\left(\mathrm{E} 2 ; 8_{1}^{+} \rightarrow 6_{1}^{+}\right){ }^{128} \mathrm{Pd}=0.035 B\left(\mathrm{E} 2 ; 2_{1}^{+} \rightarrow 0_{1}^{+}\right){ }^{130} \mathrm{Cd}
\end{aligned}
$$

which, together with the values for the conversion coefficients as obtained from the database BrIcc [46], $\alpha_{\mathrm{Cd}}(128)=0.621$ and $\alpha_{\mathrm{Pd}}(75)=3.90$, leads to the estimate $T_{1 / 2}\left({ }^{130} \mathrm{Cd} ; 8_{1}^{+}\right) \approx 9.6 \mu \mathrm{s}$, which is reasonably close to the observed value of $5.8(8) \mu \mathrm{s}$ [44]. A possible source of error in the theoretical estimate is the smallness of the $B\left(\mathrm{E} 2 ; 8_{1}^{+} \rightarrow 6_{1}^{+}\right)$value in ${ }^{128} \mathrm{Pd}$, which is sensitive to small admixtures in the wave functions.

One should be aware of the limits of validity of the simple estimates given on the basis of seniority. To illustrate this point, let us return to the example of the nickel isotopes. Besides the half-life of the $J^{\pi}=8^{+}$isomer in ${ }^{70} \mathrm{Ni}$, also the Coulomb-excitation probability of the first-excited $J^{\pi}=2^{+}$state is known in this nucleus, leading to a $B\left(\mathrm{E} 2 ; 2_{1}^{+} \rightarrow 0_{1}^{+}\right)$value of $172(28) e^{2} \mathrm{fm}^{4}$ [47]. On the basis of the seniority relation between the $B(\mathrm{E} 2)$ values for the $2_{1}^{+} \rightarrow$ $0_{1}^{+}$and $8_{1}^{+} \rightarrow 6_{1}^{+}$transitions, see the first equation of Eq. (87), a half-life $T_{1 / 2}\left({ }^{70} \mathrm{Ni} ; 8_{1}^{+}\right)=73(12)$ ns is deduced, a factor three shorter than what is observed. In other words, the $2_{1}^{+} \rightarrow 0_{1}^{+} \mathrm{E} 2$ transition is faster by a factor three than expected on the basis of seniority, indicating that the $J^{\pi}=2_{1}^{+}$state has a collective structure that goes beyond a pure $\nu 1 g_{9 / 2}$ shell.

One can push this argument further and estimate the half-life of the $J^{\pi}=$ $\left(8^{+}\right)$level in ${ }^{72} \mathrm{Ni}$. Because of the structure of the solvable $J^{\pi}=6^{+}$state with seniority $v=4$, see the second equation of Eq. (50), the $8_{1}^{+} \rightarrow 6_{1}^{+} \mathrm{E} 2$ transition arguably can be expected to be collectively enhanced as well. It should therefore be estimated from the $2_{1}^{+} \rightarrow 0_{1}^{+}$and not from the $8_{1}^{+} \rightarrow 6_{1}^{+}$ E2 transition in ${ }^{70} \mathrm{Ni}$, leading to

$$
\frac{T_{1 / 2}\left({ }^{72} \mathrm{Ni} ; 8_{1}^{+}\right)}{T_{1 / 2}\left({ }^{70} \mathrm{Ni} ; 2_{1}^{+}\right)}=\frac{1+\alpha_{\mathrm{Ni}}(1260)}{1+\alpha_{\mathrm{Ni}}(199)}\left(\frac{1260}{199}\right)^{5} \frac{B\left(\mathrm{E} 2 ; 2_{1}^{+} \rightarrow 0_{1}^{+}\right)^{70} \mathrm{Ni}}{B\left(\mathrm{E} 2 ; 8_{1}^{+} \rightarrow 6_{1}^{+}\right)^{72} \mathrm{Ni}}
$$



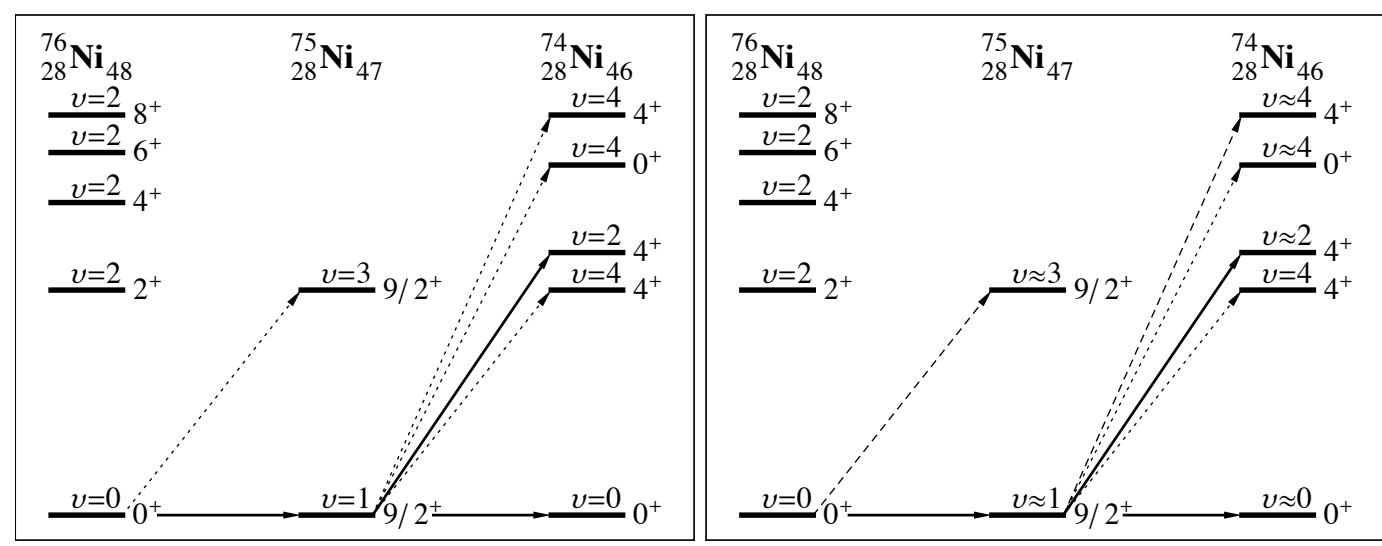

Fig. 7. Selection rules for one-particle transfer in a $j=9 / 2$ shell. In the left panel seniority is an exact quantum number for all states while in the right panel it is broken for most states, except for the two-particle states and for the four-particle $J^{\pi}=4^{+}$state with seniority $v=4$. Full lines indicate transitions allowed in one-particle transfer, dotted lines correspond to forbidden transitions and dashed lines are transitions which arise due to seniority mixing and therefore are weak but not exactly zero. Example nuclei corresponding to the two-, three- and four-particle systems are indicated on top. Energies of levels are not drawn to scale.

From the relation (see Fig. 4)

$$
B\left(\mathrm{E} 2 ; 8_{1}^{+} \rightarrow 6_{1}^{+}\right)_{72 \mathrm{Ni}}=1.08 B\left(\mathrm{E} 2 ; 2_{1}^{+} \rightarrow 0_{1}^{+}\right)_{70 \mathrm{Ni}},
$$

and the half-life $T_{1 / 2}\left({ }^{70} \mathrm{Ni} ; 2_{1}^{+}\right)=1.6(3) \mathrm{ps}$, deduced from the $B\left(\mathrm{E} 2 ; 2_{1}^{+} \rightarrow 0_{1}^{+}\right)$ value, one obtains the estimate $T_{1 / 2}\left({ }^{72} \mathrm{Ni} ; 8_{1}^{+}\right) \approx 14 \mathrm{~ns}$, which is consistent with the current upper limit of $20 \mathrm{~ns}$ [33].

\subsection{Seniority and one-nucleon transfer}

The energy spectrum of four identical particles (or holes) in a $j=9 / 2$ shell will, for any reasonable nuclear interaction, display two $J^{\pi}=4^{+}$and two $J^{\pi}=6^{+}$levels which are close in energy. Despite this closeness in energy all states retain their character. In fact, under the assumption of a pure $(9 / 2)^{4}$ configuration, the partial conservation of seniority leads to one level with exact seniority $v=4$ and the other with approximate seniority $v \approx 2$ as it mixes (but only weakly) with the other state with seniority $v=4$ at higher energy.

Partial conservation of seniority should therefore have consequences with regard to one-particle transfer. Since the seniority of a single particle is $v=1$, the selection rule associated with this reaction is $\Delta v= \pm 1$. If seniority is conserved for all eigenstates in a $j=9 / 2$ shell, the intensities of the transfer from a two- to a three-particle system and from a three- to a four-particle system are therefore as indicated in the left panel of Fig. 7: they vanish exactly 
if $\Delta v \neq \pm 1$. For clarity only the selection rules for the $J^{\pi}=4^{+}$states are indicated in Fig. 7 but the same ones are valid for the $J^{\pi}=6^{+}$states.

If, more realistically, a general and arbitrary interaction is taken in the $j=9 / 2$ shell, the intensity pattern shown in the left panel of Fig. 7 applies. As expected, since most of the levels do not carry any longer exact seniority, some of the forbidden transitions become allowed; their intensities remain small, however, since the seniority mixing is expected to be weak. More surprisingly, two of the one-particle transfer intensities remain exactly zero in spite of the seniority mixing. The explanation of these selection rules is intricate and related to the geometry of the $j=9 / 2$ shell.

Firstly, the one-particle transfer from the $J^{\pi}=9 / 2^{+}$three-particle ground state with seniority $v \approx 3$ to the second $J^{\pi}=0^{+}$four-particle state with seniority $v \approx 4$ remains exactly forbidden. The seniority mixing for the $J^{\pi}=$ $9 / 2^{+}$states is derived from the matrix

$$
\begin{aligned}
\left\langle 9 / 2_{v=1}^{+}|\hat{V}| 9 / 2_{v=1}^{+}\right\rangle & =\frac{4}{5} \nu_{0}+\frac{1}{4} \nu_{2}+\frac{9}{20} \nu_{4}+\frac{13}{20} \nu_{6}+\frac{17}{20} \nu_{8}, \\
\left\langle 9 / 2_{v=1}^{+}|\hat{V}| 9 / 2_{v=3}^{+}\right\rangle & =\frac{-65 \nu_{2}+315 \nu_{4}-403 \nu_{6}+153 \nu_{8}}{20 \sqrt{429}}, \\
\left\langle 9 / 2_{v=3}^{+}|\hat{V}| 9 / 2_{v=3}^{+}\right\rangle & =\frac{13}{132} \nu_{2}+\frac{735}{572} \nu_{4}+\frac{961}{660} \nu_{6}+\frac{459}{2860} \nu_{8} .
\end{aligned}
$$

It is seen that this mixing matrix is proportional to the one for the $J^{\pi}=0^{+}$ states given in Eq. (83). The mixings cancel as a result and the seniority selection rule remains exact.

Secondly, the one-particle transfer from the $J^{\pi}=9 / 2^{+}$ground state of the three-particle system to the solvable $J^{\pi}=4^{+}$and $J^{\pi}=6^{+}$four-particle state with seniority $v=4$ also remains exactly forbidden, despite $v=3$ admixtures in the former state. The reason in this case is that

$$
\left.\left[j^{3}\left(v=3, J_{3}=j\right) j J \mid\right\} j^{4}, v=4, \mathrm{~s}, J\right]=0,
$$

for $j=9 / 2$ and for the solvable four-particle states with $J=4$ and $6[25,26]$.

In summary, the interesting aspect of these results is that two $J^{\pi}=4^{+}$states and two $J^{\pi}=6^{+}$states are predicted to be close in energy but that they should be differently excited in a one-particle transfer reaction. To test whether these schematic predictions remain valid in a more realistic scenario, they should be compared with the results of a large-scale shell-model (LSSM) calculation. This has been done for the neutron-rich nickel isotopes with the conclusion that these characteristic features are still present in the LSSM calculation for the $J^{\pi}=6^{+}$but not for the $J^{\pi}=4^{+}$states [48]. 


\section{Conclusions}

In this paper a review of the seniority quantum number in many-body systems was given. The analysis was carried out for bosons and fermions simultaneously but was restricted to identical particles occupying a single shell (or, alternatively, particles with the same spin). The conditions of complete solvability were shown to be more restrictive than those for the conservation of seniority. The partial conservation of seniority was shown to be a peculiar property of spin-9/2 fermions but prevalent in systems of interacting bosons of any spin. Partial conservation of seniority was shown to be at the basis of the existence of seniority isomers which are frequently found in semi-magic nuclei, and to

give rise to selection rules in one-nucleon transfer reactions that have yet to be tested experimentally.

A general result of the present analysis is the proof that any system of interacting bosons with spin $\ell \leq 2$ is integrable and that its spectrum in energy is analytically available for any number of bosons. This property has been exploited in the discussion of Bose-Einstein condensates consisting of atoms with hyperfine spins $f=1$ [49] and $f=2[50,51,52]$. A more subtle property is the partial conservation of seniority in systems of interacting bosons with spin $\ell>2$. The consequences of this finding for Bose-Einstein condensates are still to be explored.

This work, elementary though it may be, paves the way for further studies which can be carried out along similar lines. Possible generalizations concern systems of (i) non-identical particles and (ii) particles distributed over several shells. Open-shell atomic nuclei provide examples of fermionic systems of type (i). The isoscalar component of the nuclear interaction, however, strongly breaks seniority and, therefore, the study of seniority in such systems has seemed irrelevant so far. Given the current interest in two-component Bose-Einstein condensates, the problem should be revisited for bosons. By considering semi-magic nuclei as examples of systems of type (ii), they can be treated more realistically than with the approach advocated in the present paper. Moreover, interesting formal questions can be explored concerning connections with generalized seniority [53] as well as with integrable RichardsonGaudin models [54]. These problems are currently under study and will be the material of the subsequent papers in this series.

\section{Acknowledgements}

Part of this work was done in collaboration with Igor Čeliković and Larry Zamick. We wish to thank Alex Brown, Ami Leviatan, Igal Talmi and John 
Wood for illuminating discussions at various stages of this work.

\section{References}

[1] G. Racah, Phys. Rev. 63 (1943) 367.

[2] G. Racah, in L. Farkas Memorial Volume, Research council of Israel, Jerusalem, 1952, p. 294.

[3] B.H. Flowers, Proc. Roy. Soc. (London) A 212 (1952) 248.

[4] A. de-Shalit and I. Talmi, Nuclear Shell Theory, Academic Press, New York, 1963.

[5] I. Talmi, Simple Models of Complex Nuclei. The Shell Model and Interacting Boson Model, Harwood, Chur, 1993.

[6] B.G. Wybourne, Classical Groups for Physicists, Wiley, New York, 1974.

[7] A.K. Kerman, Ann. Phys. (NY) 12 (1961) 300.

[8] K. Helmers, Nucl. Phys. 23 (1961) 594.

[9] A. Frank and P. Van Isacker, Algebraic Methods in Molecular and Nuclear Structure Physics, Wiley-Interscience, New York, 1994.

[10] F. Iachello, Lie Algebras and Applications, Springer-Verlag, Berlin, 2006.

[11] S. Heinze, Program ArbModel, University of Köln (unpublished).

[12] D.J. Rowe and G. Rosensteel, Phys. Rev. Lett. 87 (2001) 172501.

[13] G. Rosensteel and D.J. Rowe, Phys. Rev. C 67 (2003) 014303.

[14] J.N. Ginocchio and W.C. Haxton, in Symmetries in Science VI, edited by B. Gruber and M. Ramek, Plenum, New York, 1993.

[15] Y. Alhassid and A. Leviatan, J. Phys. A 25 (1992) L1265.

[16] A. Leviatan, Phys. Rev. Lett. 77 (1996) 818.

[17] J.E. García-Ramos, A. Leviatan and P. Van Isacker, Phys. Rev. Lett. 102 (2009) 112502.

[18] A. Leviatan, A. Novoselsky and I. Talmi, Phys. Lett. B 172 (1986) 144.

[19] P. Van Isacker, Phys. Rev. Lett. 83 (1999) 4269.

[20] A. Leviatan and P. Van Isacker, Phys. Rev. Lett. 89 (2002) 222501.

[21] I. Talmi, Nucl. Phys. A 846 (2010) 31.

[22] A. Escuderos and L. Zamick, Phys. Rev. C 73 (2006) 044302. 
[23] L. Zamick, Phys. Rev. C 75 (2007) 064305.

[24] P. Van Isacker and S. Heinze, Phys. Rev. Lett. 100 (2008) 052501.

[25] L. Zamick and P. Van Isacker, Phys. Rev. C 78 (2008) 044327.

[26] C. Qi, Phys. Rev. C 83 (2011) 014307.

[27] H. Weyl, The Classical Groups, Princeton University Press, Princeton, 1939.

[28] A. Gheorghe and A.A. Raduta, J. Phys. A: Math. Gen. 37 (2004) 10951.

[29] P. Walker and G. Dracoulis, Nature 399 (1999) 35.

[30] J.M. Jaklevic, C.M. Lederer and J.M. Hollander, Phys. Lett. B 29 (1969) 179.

[31] H. Grawe, M. Górska, M. Lipoglavšek, J. Nyberg, R. Grzywacz, M. Lewitowicz, K. Rykaczewski, K.H. Maier and R. Schubart, Prog. Part. Nucl. Phys. 38 (1997) 15.

[32] M. Sawicka et al., Phys. Rev. C 68 (2003) 044304.

[33] C.J. Chiara et al., Phys. Rev. C 84 (2011) 037304.

[34] H. Grawe et al., Nucl. Phys. A 704, 211c (2002).

[35] J.K. Tuli, Nucl. Data Sheets 103 (2004) 389.

[36] C. Mazzocchi et al., Phys. Lett. B 622 (2005) 45.

[37] A.F. Lisetskiy, B.A. Brown, M. Horoi and H. Grawe, Phys. Rev. C 70 (2004) 044314 .

[38] C.M. Baglin, Nucl. Data Sheets 91 (2000) 423.

[39] W.J. Mills, J.J. Ressler, R.A.E. Austin, R.S. Chakrawarthy, D.S. Cross, A. Heinz, E.A. McCutchan and M.D. Strange, Phys. Rev. C 75 (2007) 047302.

[40] D. Abriola and A.A. Sonzogni, Nucl. Data Sheets 109 (2008) 2501.

[41] B. Singh and Z. Hu, Nucl. Data Sheets 98 (2003) 335.

[42] P. Van Isacker, J. Phys.: Conf. Series 322 (2011) 012003.

[43] A. Gottardo et al., Phys. Rev. Lett. 109 (2012) 162502.

[44] H. Watanabe et al., Phys. Rev. Lett. 111 (2013) 152501.

[45] A. Jungclaus et al., Phys. Rev. Lett. 99 (2007) 132501.

[46] T. Kibédi, T.W. Burrows, M.B. Trzhaskovskaya, P.M. Davidson and C.W. Nestor Jr., Nucl. Instr. Meth. A 589 (2008) 202.

[47] O. Perru et al., Phys. Rev. Lett. 96 (2006) 232501.

[48] P. Van Isacker and I. Celiković, Proc. XIVth Int. Symp. on Capture GammaRay Spectroscopy and Related Topics, P.E. Garrett and B. Hadinia, Eds., World Scientific, Singapore, 2013. 
[49] C.K. Law, H. Pu and N.P. Bigelow, Phys. Rev. Lett. 81 (1998) 5257.

[50] P. Van Isacker and S. Heinze, J. Phys. A: Math. Theor. 40 (2007) 14811.

[51] S. Uchino, T. Otsuka and M. Ueda, Phys. Rev. A 78 (2008) 023609.

[52] Y.Z. He and C.G. Bao, Phys. Rev. A 84 (2011) 063620.

[53] I. Talmi, Nucl. Phys. A 172 (1971) 1.

[54] J. Dukelsky, S. Pittel and G. Sierra, Rev. Mod. Phys. 76 (2004) 643. 